ICTP-PH-TH/2012-xyz

\title{
On higher derivative corrections to Wess-Zumino and Tachyonic actions in type II super string theory
}

\author{
Ehsan Hatefi1 \\ International Centre for Theoretical Physics, Strada Costiera 11, Trieste, Italy
}

\begin{abstract}
We evaluate in detail the string scattering amplitude to compute different interactions of two massless scalars, one tachyon and one closed string RamondRamond field in type II super string theory. In particular we find two scalar field and two tachyon couplings to all orders of $\alpha^{\prime}$ up to on-shell ambiguity. We then obtain the momentum expansion of this amplitude and apply this infinite number of couplings to actually check that the infinite number of tachyon poles of S-matrix element of this amplitude for the $p=n$ case (where $p$ is the spatial dimension of a $\mathrm{D}_{p}$-brane and $n$ is the rank of a Ramond-Ramond field strength ) to all orders of $\alpha^{\prime}$ is precisely equal to the infinite number of tachyon poles of the field theory. In addition to confirming the couplings of closed string Ramond-Ramond field to the world-volume gauge field and scalar fields including commutators, we also propose an extension of the Wess-Zumino action which naturally reproduces these new couplings in field theory such that they could be confirmed with direct S-matrix computations. Finally we show that the infinite number of massless poles and contact terms of this amplitude for the $p=n+1$ case can be reproduced by Chern-Simons, higher derivative corrections of the Wess-Zumino and symmetrized trace tachyon DBI actions.
\end{abstract}

\footnotetext{
${ }^{1}$ E-mail:ehatefi@ictp.it
} 


\section{Introduction}

$\mathrm{D}_{p}$-branes must be regarded as the sources of Ramond-Ramond $(p+1)$-form fields in type II super string theories [1]. Their perturbative excitations should be understood as fundamental open string states on their world volume. More details can be found in 2, 3]. Having taken into account $\mathrm{N}$ coincident D-branes, we must have $\mathrm{U}(\mathrm{N})$ non abelian symmetry [2] Notice that the bosonic action was derived in [4, 5].

The low energy action representing D-branes' dynamics defined as Born-Infeld action :

$$
S_{B I}=-T_{p} \int d^{p+1} \sigma \operatorname{STr}\left(e^{-\phi} \sqrt{-\operatorname{det}\left(P\left[E_{a b}+E_{a i}\left(Q^{-1}-\delta\right)^{i j} E_{j b}\right]+\lambda F_{a b}\right) \operatorname{det}\left(Q^{i}{ }_{j}\right)}\right),
$$

with

$$
E_{a b}=G_{a b}+B_{a b} \quad, \quad Q^{i}{ }_{j} \equiv \delta^{i}{ }_{j}+i \lambda\left[\Phi^{i}, \Phi^{k}\right] E_{k j},
$$

For more details see [4, 6]. Remember different couplings in this action for BPS branes have been shown to be consistent with disk level amplitudes in super string theory [6, 7, 8, 9]. The important point should be made is that derivatives of the field strength of the gauge field, and the second and higher derivatives of the scalars must be embedded to this action(see [7, 8]).

We should introduce the Wess-Zumino action as well. This action in the presence of closed string RR field (showed by $C$ )makes sense as follows [1, 10]

$$
S_{W Z}=\mu_{p} \int \operatorname{STr}\left(P\left[e^{i \lambda i_{\Phi} i_{\Phi}}\left(\sum C^{(n)}\right)\right] e^{\lambda F}\right) .
$$

Note that we have set

$$
G_{\mu \nu}=\eta_{\mu \nu}, \quad B_{\mu \nu}=\Phi=0
$$

so one may believe that we are working on flat space background. In our conventions $\lambda=2 \pi \alpha^{\prime}$. We want to begin with non-supersymmetric D-branes in type II theory where $p$ is odd for IIA and even for IIB. In the spectrum of a non-BPS D-brane in type II theory, there exists a tachyonic state (indicated by $T$ ), a massless gauge field (showed by $A_{a}$ ) and some massless scalars $\phi^{i}$ and some fermions (for example see [11]). All scalars might express transverse oscillations of the brane.

Now the open string tachyon must be condensed to a kink to produce a stable $D_{p-1^{-}}$ brane. To study brane production in detail see [12, 13, 14]. It is worth mentioning the point that unstable branes first have been used in checking some duality conjectures [15]. 
Based on some concrete points [16], one might expect that the effective theory of nonBPS branes should involve tachyon and massless states within itself. Therefore the action for non-BPS branes is constructed from two parts such that

$$
S_{n o n-B P S}=S_{D B I}+S_{W Z}
$$

Now tachyon must get involved in Dirac-Born-Infeld and Wess-Zumino effective actions. Having done boundary string field theory (BSFT) method, we may talk about these effective actions, see [17, 18, for further details. Due to some efforts [17, 18, 19] tachyons have already been taken into account in the WZ effective action using the superconnection of noncommutative geometry [20]. Concerning super connection [20], one can write down the WZ action as follows

$$
S_{W Z}=\mu_{p}^{\prime} \int_{\Sigma_{(p+1)}} C \wedge \mathrm{S} \operatorname{Tr} e^{i 2 \pi \alpha^{\prime} \mathcal{F}},
$$

where $\Sigma_{(p+1)}$ is the world volume, $\mu_{p}^{\prime}$ is the RR charge of branes. The curvature of super connection first has been derived for brane anti brane system [21] then the method was extended to include non-BPS branes [22] and it is obtained as

$$
i \mathcal{F}=\left(\begin{array}{cc}
i F-\beta^{\prime 2} T^{2} & \beta^{\prime} D T \\
\beta^{\prime} D T & i F-\beta^{\prime 2} T^{2}
\end{array}\right),
$$

One must emphasize that superconnection's structure in the WZ action first has been achieved with the direct S-matrix method as appeared in [19]. The tensor structure of this object is such that the definitions of field strength of the gauge field and covariant derivative of tachyon are

$$
F=\frac{1}{2} F_{a b} d x^{a} \wedge d x^{b} \quad, D T=\left(\partial_{a} T-i\left[A_{a}, T\right]\right) d x^{a}
$$

where $\beta^{\prime}$ is a normalization tachyon which is constant with dimension $\left(1 / \sqrt{\alpha^{\prime}}\right)^{2}$. It is shown that $\beta^{\prime}=\frac{1}{\pi} \sqrt{\frac{6 \ln (2)}{\alpha^{\prime}}}[23$. More consistency of the WZ action with other S-matrix elements can be seen in [21, 23]. Applying the expansion for the exponential term in the WZ action (5), one gets 22 .

$$
\begin{gathered}
\mu_{p}^{\prime}\left(2 \pi \alpha^{\prime}\right) C \wedge \operatorname{Str} i \mathcal{F}=2 \beta^{\prime} \mu_{p}^{\prime}\left(2 \pi \alpha^{\prime}\right) \operatorname{Tr}\left(C_{p} \wedge D T\right), \\
\frac{\mu_{p}^{\prime}}{2 !}\left(2 \pi \alpha^{\prime}\right)^{2} C \wedge \operatorname{Str} i \mathcal{F} \wedge i \mathcal{F}=2 \beta^{\prime} \mu_{p}^{\prime}\left(2 \pi \alpha^{\prime}\right)^{2} \operatorname{Tr}\left(C_{p-2} \wedge D T \wedge F\right) .
\end{gathered}
$$

\footnotetext{
${ }^{2} \alpha^{\prime}=l_{s}^{2}$ and $l_{s}$ becomes string length scale.
} 
To study the effective action of non-BPS branes we would like to proceed with the second approach, which is the S-matrix formalism. In this method one must include kinetic term of the tachyon in the DBI part [24]; however, due to considering internal degrees of freedom or internal Chan-Paton factors belonging to non-BPS branes this action gets modified [23, 25].

Morever, according to the S-matrix method, one subtlety around the unstable point of the tachyon DBI does exist. To be more specific, all massless fields must carry an identity internal CP matrix, while tachyon in the (0)-picture has to carry $\sigma_{1}$ and it does carry the $\mathrm{CP}$ matrix of $\sigma_{2}$ in the (-1)-picture. Note also that the picture changing operator carries $\sigma_{3}$ internal CP matrix [26]. Notice that, this important point has not been confirmed yet around the stable point of the tachyon action.

The outline of the paper is as follows. In section 3 we are going to compute in detail a tree-level physically four point (technically five point) string scattering amplitude including one RR, two scalar field and one tachyon vertex operators in the world volume of type II super string theory. The world-volume theory might be rewritten in terms of an infinite number of the derivatives of the fields as one of the main goals of the paper is indeed obtaining those infinite numbers of the couplings between two tachyons and two scalar fields to all orders of $\alpha^{\prime}$. Keeping in mind that once the world volume fields vary so slowly, we must get the usual effective theory by reducing the higher derivative theories.

In section 4 we carry out the momentum expansion whose leading order terms must be consistent with field theory. On the other hand, the rest of the terms which are non leading terms must correspond to the higher derivative corrections of tachyon DBI and WessZumino effective actions. We conjecture that we found a unique momentum expansion for all four point functions including one RR, one tachyon and two massless open strings which can be either two gauge fields or two scalar fields. We guess this happens also in the amplitude of one RR, one tachyon, one gauge field and one scalar field but it has not been checked yet so we postpone it for future work [27]. In section 5 we talk about effective field theory on the world volume of brane. In section 5.1 using symmetrized trace tachyon DBI action we reproduce the first tachyon pole in field theory for $p+1=n$ case, where $p$ is the spatial dimension of a $\mathrm{D}_{p}$-brane and $n$ is the rank of a $\mathrm{RR}$ field strength.

To obtain the infinite number of tachyon poles for this case, one needs to know the 
higher derivative couplings of two scalar fields and two tachyons up to all orders of $\alpha^{\prime}$. Applying the string computations and making use of some how T-duality transformation in section 6 we find higher derivative couplings of two scalar fields and two tachyons up to all orders of $\alpha^{\prime}$ within on-shell ambiguity. Then using the infinite number of the couplings obtained in field theory we will show that the infinite number of tachyon poles of this Smatrix element are exactly reproduced to all orders of $\alpha^{\prime}$. In section 7.1 we obtain all infinite numbers of massless poles in field theory for $p=n+1$ case. In order to get consistent results between string theory and field theory we propose an extension of the Wess-Zumino action and discover some sort of new couplings which could be confirmed by direct S-matrix computations.

We also generate all infinite contact terms of this amplitude. Eventually we end up with a discussion, mention our results, and give some hints for future directions. Appendix A consists of some information about doubling trick in II super string theory, some useful comments on conformal field theory propagators, and some correlation functions including two spin operators and some number of fermion fields and/or currents. Appendix B includes some useful integrals for five point functions. Let us address our notation.

$\mu, \nu$ represent the entire space-time dimensions and $\mu, \nu=0, \ldots, 9$ and $a, b, c$ indices show world-volume space $a, b, c=0,1, \ldots, p$ and finally $i, j$ indices indicate transverse space, $i, j=$ $p+1, \ldots, 9$.

\section{Remarks on Scattering amplitude}

The S-Matrix technique is a very important tool in super string theory to actually discover new couplings between mixed combinations of open and closed strings. Therefore corrections in field theory might have been obtained in $\alpha^{\prime}$ by standard scattering amplitude methods. To describe scattering of closed strings with open strings see [6, 28]. To observe one of the important aspects of D-brane physics we refer the reader to [29]. In order to follow the scattering argument and its applications, it is worth looking at [30].

\subsection{Motivations}

Pursuing scattering amplitudes has been one of the most active fields during the recent years [31]. The (Britto,Cachazo,Feng and Witten) BCFW recursion relations were derived by taking into account the fact that an $n$-point tree level amplitude is expressed in terms 
of the rational function of all external momenta. Thus by applying analytic continuation of all momenta to an entire complex plane, the amplitude would be constructed by its poles. Some more attempts have been made in [32, 33] which help one to realize which kinds of theories should be determined by the BCFW relations.

The string BCFW relations have been investigated in [34, 35] for the scattering amplitude of external open string tachyons.

Because of some kinematic reasons it is not possible to embed their efforts within our formalism which is perturbative string calculations. We will notice these arguments in the section of momentum expansion. In fact our computations make sense just in the presence of a constant value of RR 's momentum which is indeed $p_{a} p^{a} \rightarrow \frac{1}{4}$, and it is going to be the key point in our calculations.

The second motivation for studying unstable objects perhaps is realizing properties of string theory in time-dependent backgrounds [36, 37]. Open string tachyons are showing us the instability of the processes, which we are interested in. A. Sen in [38] has shown that tachyon DBI action [39] might be able to represent decay non-BPS D-branes [40] around the stable point of tachyon potential.

Another motivation to follow unstable branes is in fact studying spontaneous chiral symmetry breaking in some holographic patterns in QCD [41]. In order to have a formalism for inflation (of course in string theory), one may use D-brane anti D-brane effective action[21] gets divided along an extra dimension [42]. In this formalism the brane separation has to play inflaton's role. When this distance is smaller than the string scale, the open strings stretching between them will become two real tachyon modes and they may condense [43, 44, 45]. Indeed the negative energy density of the tachyon field which is condensed cancels the positive energy density of the brane anti brane and gives us a $D_{p-2}$-brane with a finite tension [46]. Then inflation ends and finally the energy of inflaton will decay to the particles in the Standard model [47]. To follow some of the applications of this action in cosmology references [48] may be worth referencing.

Having read the boundary conformal field theory (BCFT), some of the results such as producing a pressure less gas with nonvanishing energy density at the end of tachyon condensation [49] may be obtained from this action at the minimum of the tachyon potential. Although the higher derivative terms might have a significant role at the top of the tachyon potential, they have small effects at the minimum of the potential. Note that the higher 
derivative terms are not involved in the BSFT action. Thus, the action which was obtained through S-matrix elements at the top of the potential may indeed represent the effective action of string theory at the minimum of the tachyon potential. Notice that the action is also consistent with T-duality rules.

On the other hand, the effective action for brane-antibrane [50] based on the S-matrix elements computations is found in [21] to be

$$
S_{D B I}=-T_{p} \int d^{p+1} \sigma \mathrm{S} \operatorname{Tr}\left(V(\mathcal{T}) \sqrt{-\operatorname{det}\left(\eta_{a b}+2 \pi \alpha^{\prime} F_{a b}+2 \pi \alpha^{\prime} D_{a} \mathcal{T} D_{b} \mathcal{T}\right)}\right)
$$

STr means symmetric trace for all matrices including $F_{a b}, D_{a} \mathcal{T}$ and $\mathcal{T}$ in the potential. To see more information and the explicit form of these matrices see [21]. The tachyon potential that does make an exact result with S-matrix computations has an expansion as:

$$
V(|T|)=1+\pi \alpha^{\prime} m^{2}|T|^{2}+\frac{1}{2}\left(\pi \alpha^{\prime} m^{2}|T|^{2}\right)^{2}+\cdots
$$

which is consistent expansion in comparing with the tachyon potential in BSFT which has $V(|T|)=e^{\pi \alpha^{\prime} m^{2}|T|^{2}}$ potential form [51]. It is also consistent with the sigma model effective action [52]. Note that $m^{2}$ is tachyon's mass square and $m^{2}=-1 /\left(2 \alpha^{\prime}\right)$.

Having expanded the square root, and renormalized $\frac{1}{2} \pi T^{2} \rightarrow 2 T^{2}$, we get

$$
S=-T_{p} e^{-T^{2}}\left(1+4 \alpha^{\prime} D_{a} T D^{a} T+\cdots\right)
$$

which is precisely the term coming from BSFT action suggested in [53] .

Therefore using the S-matrix method, we are able to find out either tachyon action around the unstable point of non-BPS D-branes or D-brane anti D-brane where the higher derivatives of tachyon are indeed important. So not only is it really interesting to study them but also worth it to obtain their general form to all orders of $\alpha^{\prime}$.

The existence of the coupling $\operatorname{Tr}\left(C_{p-2} \wedge F \wedge D T\right)$ has already been checked in [23] by working out in detail the disk level S-matrix element of one RR field, one tachyon and two gauge fields in the world volume of a single non-BPS brane. In this paper among other things, we find some new contact interactions of the form $\operatorname{Tr}\left(C_{p-2} \wedge D T \wedge D \phi^{i} \wedge D \phi_{i}\right)$, $\operatorname{Tr}\left(C_{p} \wedge D T \phi^{i} \phi_{i}\right), \operatorname{Tr}\left(\partial_{a_{0}} T\left[\Phi^{i}, \Phi^{j}\right]\right) C_{j i a_{1} \cdots a_{p}}^{(p+2)}\left(\varepsilon^{v}\right)^{a_{0} \cdots a_{p}}$ and some other couplings which can be confirmed just by S-matrix computations and fix their coefficients using the S-matrix elements of one RR field, one tachyon and two scalar fields. 


\section{The Scattering amplitude between one closed string RR, two scalar fields and one tachyon}

By making use of the conformal field theory techniques, we might carry out the string scattering amplitude to find out all couplings of one closed string RR field in the bulk to two open string scalar fields and one open string tachyon on the world-volume of a single non-BPS D-brane with flat empty space background. To compute a S-matrix element, one must fix the picture of the vertex operators .

Knowing the fact that the total super ghost charge for disk level amplitude must be -2, we will choose the vertex operators accordingly. Concerning string duality, we map the disk to the upper half plane thus the boundary of the disk becomes the real axis. Therefore, the closed string vertex operator should be inserted at the middle and all open string vertex operators must be put at the boundary of the disk world-sheet. It is fair to say that some efforts in order for obtaining the string scattering amplitudes at tree level in both BPS and non BPS formalism have been made [6, 17, 8, 9, 19, 21, 23, 54]. The external states will be appeared in our amplitude as the following:

$$
\begin{aligned}
\text { tachyon } & T, k_{3}, \\
\text { transverse scalars : } & \Phi_{1}^{i}, k_{1}, \\
& \Phi_{2}^{j}, k_{2}, \\
\operatorname{RR}(p+1) \text {-form : } & C^{p+1}, p .
\end{aligned}
$$

We also define

$$
s=-\frac{\alpha^{\prime}}{2}\left(k_{1}+k_{3}\right)^{2}, \quad t=-\frac{\alpha^{\prime}}{2}\left(k_{1}+k_{2}\right)^{2}, \quad u=-\frac{\alpha^{\prime}}{2}\left(k_{2}+k_{3}\right)^{2} .
$$

Applying momentum conservation along the world volume of the brane, we get the following relation

$$
s+t+u=-p^{a} p_{a}-\frac{1}{4}
$$

Remember the fact that the vertex operators of a non-BPS D-brane must carry internal degrees of freedom or the internal Chan-Paton(CP) matrix [11]. In the other words in order to distinguish the form of closed string vertex operators of non BPS branes from their form in the brane-antibrane system an internal CP matrix is needed. By setting tachyon to zero, we may conclude that both the effective field theory of brane-antibrane and non-BPS 
branes must be reduced to effective field theory of just BPS branes. Therefore we come to the key point which is imposing an identity internal CP matrix for all massless fields in both brane-antibrane and non-BPS branes' formalism.

As an example, the RR field in effective theory of brane-antibrane does include an identity matrix. This is related to the fact that by setting tachyon to zero the Wess-Zumino action of brane-antibrane does go back to the Wess-Zumino action of two BPS branes. We devote this identity internal CP matrix to RR, gauge field and scalar field vertex operators in the (0)-picture. In [26] it has been shown that the picture changing operator does carry internal $\mathrm{CP}$ matrix $\sigma_{3}$ and so in the (-1)-picture, the internal $\mathrm{CP}$ matrix of RR vertex operator in the brane-antibrane system and gauge and scalar (in both non-BPS brane and brane-antibrane system) is not identity any more and in fact it is $\sigma_{3}$.

Notice that in non-BPS branes, there must be an extra factor of $\sigma_{1}$ in the RR vertex operator [11, 22], so the RR vertex operator of a non-BPS brane in the (0)-picture must involve the internal $\mathrm{CP}$ matrix $\sigma_{1}$. We are going to address this $\mathrm{CP}$ matrix to tachyon vertex operator in (0)-picture as well. Therefore by applying the picture changing operator to $R R$ in the (0)-picture we reach the point that $R R$ vertex operator in the (-1)-picture in the non-BPS formalism has to carry the internal matrix $\sigma_{3} \sigma_{1}$. Finally by the same argument we conclude that the internal CP matrix of the tachyon in the (-1)-picture is $\sigma_{2}$.

Thus, the S-matrix element of one RR field, two scalar fields and one tachyon in the world volume of a single non-BPS D-brane in super string theory may be given with :

$$
\mathcal{A}^{\phi \phi T C} \sim \sum_{\text {non-cyclic }} \int d x_{1} d x_{2} d x_{3} d z d \bar{z}\left\langle V_{\phi}^{(0)}\left(x_{1}\right) V_{\phi}^{(0)}\left(x_{2}\right) V_{T}^{(0)}\left(x_{3}\right) V_{R R}^{(-2)}(z, \bar{z})\right\rangle
$$

The internal CP factor is $\operatorname{Tr}\left(\sigma_{1} I I \sigma_{1}\right)=2$ for all permutations of the scalar fields. Note that in the non-BPS system both the Ramond-Ramond and tachyon in the (-2)-picture do carry $\sigma_{1}$ internal $\mathrm{CP}$ matrix and this is related to the point that the amplitude of $C T A$ makes sense in the world volume of non-BPS branes. However by taking into account the amplitude of CTT in the world volume of brane-antibrane one comes over to the CP factor of $\mathrm{RR}$ in the (-2)-picture as the identity $\mathrm{CP}$ matrix in this system. It is indeed so easy to compute this amplitude by putting the RR and tachyon in the(-1)-picture so that

$$
\mathcal{A}^{\phi \phi T C} \sim \sum_{\text {non-cyclic }} \int d x_{1} d x_{2} d x_{3} d z d \bar{z}\left\langle V_{\phi}^{(0)}\left(x_{1}\right) V_{\phi}^{(0)}\left(x_{2}\right) V_{T}^{(-1)}\left(x_{3}\right) V_{R R}^{(-1)}(z, \bar{z})\right\rangle .
$$


The CP factor now becomes $\operatorname{Tr}\left(\sigma_{3} \sigma_{1} I I \sigma_{2}\right)=2 i$ for 123 and 132 orderings. Thus to investigate the S-matrix element (12), which is much more difficult than (13), we do computations of the amplitude of (13) and finally multiply a coefficient of $(-i)$ in the final result.

In general the form of vertex operators in (13) is written down as 3

$$
\begin{aligned}
V_{T}^{(-1)}(y) & =e^{-\phi(y)} e^{2 i k \cdot X(y)} \lambda \otimes \sigma_{2}, \\
V_{\phi}^{(0)}(x) & =\xi_{i}\left(\partial X^{i}(x)+2 i q \cdot \psi \psi^{i}(x)\right) e^{2 i q \cdot X(x)} \lambda \otimes I, \\
V_{R R}^{(-1)}(z, \bar{z}) & =\left(P_{-} H_{(n)} M_{p}\right)^{\alpha \beta} e^{-\phi(z) / 2} S_{\alpha}(z) e^{i p \cdot X(z)} e^{-\phi(\bar{z}) / 2} S_{\beta}(\bar{z}) e^{i p \cdot D \cdot X(\bar{z})} \otimes \sigma_{3} \sigma_{1}, \\
V_{\phi}^{-2}(x) & =e^{-2 \phi} V_{\phi}^{0}(x),
\end{aligned}
$$

where $k, q, p$ are the momenta of tachyon, scalar field and closed string RR field accordingly. Notice that the momentum of open strings has to be constrained to be inside of the world volume. The on-shell condition for the tachyon is $k^{2}=\frac{1}{2 \alpha^{\prime}}$ and for the $\mathrm{RR}$ and scalar it is $p^{2}=q^{2}=0$. The physical state condition for the massless scalar and $\mathrm{RR}$ is $k . \xi=0$ and $p_{i}^{\mu} \epsilon_{i \mu \mu_{3} \ldots \mu_{n}}=0$. Also $\lambda$ must be regarded as an external CP matrix which should be in the $U(N)$ gauge group. The definition of projection operator is $P_{-}=\frac{1}{2}\left(1-\gamma^{11}\right)$. Throughout of the paper we work with the full $32 \times 32$ Dirac matrices in ten dimensions of space-time. The definition of the RR field strength is

$$
H_{(n)}=\frac{a_{n}}{n !} H_{\mu_{1} \ldots \mu_{n}} \gamma^{\mu_{1}} \ldots \gamma^{\mu_{n}},
$$

with $n=2,4$ for type IIA and $n=1,3,5$ for type IIB. $a_{n}=i$ for IIA and $a_{n}=1$ for IIB theory. The spin indices must be raised with the charge conjugation matrix such that

$$
\left(P_{-} H_{(n)}\right)^{\alpha \beta}=C^{\alpha \delta}\left(P_{-} H_{(n)}\right)_{\delta}^{\beta}
$$

In order to deal with standard holomorphic conformal field theory propagators on the boundary of world sheet we might use the doubling trick (see Appendix A for more details). Implementing this trick, we are allowed to use just the standard correlators for the worldsheet fields $X^{\mu}, \psi^{\mu}, \phi$ as follows

$$
\begin{aligned}
\left\langle X^{\mu}(z) X^{\nu}(w)\right\rangle & =-\eta^{\mu \nu} \log (z-w), \\
\left\langle\psi^{\mu}(z) \psi^{\nu}(w)\right\rangle & =-\eta^{\mu \nu}(z-w)^{-1} \\
\langle\phi(z) \phi(w)\rangle & =-\log (z-w) .
\end{aligned}
$$

\footnotetext{
${ }^{3}$ In string side, we used to set $\alpha^{\prime}=2$.
} 
We refer the interested reader to see Appendix A of the paper for applying the doubling trick, for working out with the standard holomorphic correlators, and for finding various correlation functions including spin operators, fermions and currents .

Note also that to simplify our computations we introduce $x_{4} \equiv z=x+i y$ and $x_{5} \equiv$ $\bar{z}=x-i y$, thus the amplitude for 123 ordering will be written as

$$
\begin{aligned}
\mathcal{A}^{\phi \phi T C} \sim & \int d x_{1} d x_{2} d x_{3} d x_{4} d x_{5}\left(P_{-} H_{(n)} M_{p}\right)^{\alpha \beta} \xi_{1 i} \xi_{2 j} x_{45}^{-1 / 4}\left(x_{34} x_{35}\right)^{-1 / 2}\left(I_{1}+I_{2}+I_{3}+I_{4}\right) \\
& \times \operatorname{Tr}\left(\lambda_{1} \lambda_{2} \lambda_{3}\right) \operatorname{Tr}\left(I I \sigma_{2} \sigma_{3} \sigma_{1}\right),
\end{aligned}
$$

with $x_{i j}=x_{i}-x_{j}$ and

$$
\begin{aligned}
I_{1}= & <: \partial X^{i}\left(x_{1}\right) e^{2 i k_{1} \cdot X\left(x_{1}\right)}: \partial X^{j}\left(x_{2}\right) e^{2 i k_{2} \cdot X\left(x_{2}\right)}: e^{2 i k_{3} \cdot X\left(x_{3}\right)}: e^{i p \cdot X\left(x_{4}\right)}: e^{i p \cdot D \cdot X\left(x_{5}\right)}:> \\
& \times<: S_{\alpha}\left(x_{4}\right): S_{\beta}\left(x_{5}\right):> \\
I_{2}= & <: \partial X^{i}\left(x_{1}\right) e^{2 i k_{1} \cdot X\left(x_{1}\right)}: e^{2 i k_{2} \cdot X\left(x_{2}\right)}: e^{2 i k_{3} \cdot X\left(x_{3}\right)}: e^{i p \cdot X\left(x_{4}\right)}: e^{i p \cdot D \cdot X\left(x_{5}\right)}:> \\
& \times<: S_{\alpha}\left(x_{4}\right): S_{\beta}\left(x_{5}\right): 2 i k_{2 b} \psi^{b} \psi^{j}\left(x_{2}\right):>, \\
I_{3}= & <: e^{2 i k_{1} \cdot X\left(x_{1}\right)}: \partial X^{j}\left(x_{2}\right) e^{2 i k_{2} \cdot X\left(x_{2}\right)}: e^{2 i k_{3} \cdot X\left(x_{3}\right)}: e^{i p \cdot X\left(x_{4}\right)}: e^{i p . D \cdot X\left(x_{5}\right)}:> \\
& \times<: S_{\alpha}\left(x_{4}\right): S_{\beta}\left(x_{5}\right): 2 i k_{1 a} \psi^{a} \psi^{i}\left(x_{1}\right):> \\
I_{4}= & <: e^{2 i k_{1} \cdot X\left(x_{1}\right)}: e^{2 i k_{2} \cdot X\left(x_{2}\right)}: e^{2 i k_{3} \cdot X\left(x_{3}\right)}: e^{i p \cdot X\left(x_{4}\right)}: e^{i p \cdot D \cdot X\left(x_{5}\right)}:> \\
& \times<: S_{\alpha}\left(x_{4}\right): S_{\beta}\left(x_{5}\right): 2 i k_{1 a} \psi^{a} \psi^{i}\left(x_{1}\right): 2 i k_{2 b} \psi^{b} \psi^{j}\left(x_{2}\right):>.
\end{aligned}
$$

Concerning the correlators corresponding to bosonic fields in Appendix A, one could compute all correlators of $X$. To find out the correlator of world sheet fermions $(\psi s)$ with two spin operators (coming from RR sector), we may use Wick-like rule [21, 55] (see also Appendix A).

Wick-like rule might be also generalised to obtain the correlation function of two spin operators and a number of currents and fermion fields [6].

There are two subtleties in applying the formula (2) for currents. The first one is that one should not take into account the Wick-like contraction for two fermion fields within one current. The second one is that one has to pay attention to all minus signs which are coming from fermion propagators once we want to write down Wick-like contraction of two fermion fields in which they belong to two different currents [6]. This point is playing the key role in all straightforward but tedious computations. Considering those issues, one gets the old results in the presence and absense of current as they have already been checked in [23]. 
When there are two currents, the formula (2) gives a more complicated result. However, here there is no correlation between the transverse fields and world volume fields so we end up some how with simple result as follows

$$
\begin{aligned}
I_{4}^{\prime}= & <: S_{\alpha}\left(x_{4}\right): S_{\beta}\left(x_{5}\right): \psi^{a} \psi^{i}\left(x_{1}\right): \psi^{b} \psi^{j}\left(x_{2}\right):> \\
= & \frac{1}{4} x_{45}^{3 / 4}\left(x_{14} x_{15} x_{24} x_{25}\right)^{-1}\left\{\left(\Gamma^{j b i a} C^{-1}\right)_{\alpha \beta}+2 \frac{\operatorname{Re}\left[x_{14} x_{25}\right]}{x_{12} x_{45}}\left[\eta^{a b}\left(\Gamma^{j i} C^{-1}\right)_{\alpha \beta}+\eta^{i j}\left(\Gamma^{b a} C^{-1}\right)_{\alpha \beta}\right]\right. \\
& \left.+4\left(\frac{\operatorname{Re}\left[x_{14} x_{25}\right]}{x_{12} x_{45}}\right)^{2}\left(-\eta^{a b} \eta^{i j}\right) C_{\alpha \beta}^{-1}\right\},
\end{aligned}
$$

Having performed the $X$ correlators and replacing (16) in (15), one may write the amplitude as

$$
\begin{aligned}
\mathcal{A}^{\phi \phi T C} \sim & \int d x_{1} d x_{2} d x_{3} d x_{4} d x_{5}\left(P_{-} H_{(n)} M_{p}\right)^{\alpha \beta} I \xi_{1 i} \xi_{2 j} x_{45}^{-1 / 4} x_{34}^{-1 / 2} x_{35}^{-1 / 2} \\
& \times\left(x_{45}^{-5 / 4} C_{\alpha \beta}^{-1}\left(-\eta^{i j} x_{12}^{-2}+a_{1}^{i} a_{2}^{j}\right)+a_{1}^{i}\left(a_{3}^{j}\right)_{\alpha \beta}+a_{2}^{j}\left(a_{4}^{i}\right)_{\alpha \beta}-4 k_{1 a} k_{2 b} I_{4}^{\prime}\right),
\end{aligned}
$$

where $I_{4}^{\prime}$ is given in (16) and

$$
\begin{aligned}
I & =\left|x_{12}\right|^{4 k_{1} \cdot k_{2}}\left|x_{13}\right|^{4 k_{1} \cdot k_{3}}\left|x_{14} x_{15}\right|^{2 k_{1} \cdot p}\left|x_{23}\right|^{4 k_{2} \cdot k_{3}}\left|x_{24} x_{25}\right|^{2 k_{2} \cdot p}\left|x_{34} x_{35}\right|^{2 k_{3} \cdot p}\left|x_{45}\right|^{p . D . p}, \\
a_{1}^{i} & =-i p^{i}\left(\frac{x_{45}}{x_{14} x_{15}}\right), \\
a_{2}^{j} & =-i p^{j}\left(\frac{x_{45}}{x_{24} x_{25}}\right), \\
\left(a_{3}^{j}\right)_{\alpha \beta} & =i k_{2 b} x_{45}^{-1 / 4}\left(\Gamma^{j b} C^{-1}\right)_{\alpha \beta}\left(x_{24} x_{25}\right)^{-1}, \\
\left(a_{4}^{i}\right)_{\alpha \beta} & =i k_{1 a} x_{45}^{-1 / 4}\left(\Gamma^{i a} C^{-1}\right)_{\alpha \beta}\left(x_{14} x_{15}\right)^{-1} .
\end{aligned}
$$

As the first check of our computations, the amplitude is now invariant under SL(2,R) transformations. One may try to cancel the volume of the conformal Killing group by fixing the positions of the three open strings. So to set gauge fixing of this symmetry we used to fix the position of the open strings as

$$
x_{1}=0, \quad x_{2}=1, \quad x_{3} \rightarrow \infty, \quad d x_{1} d x_{2} d x_{3} \rightarrow x_{3}^{2}
$$

Note that different fixing for position of open strings will give rise to different ordering in the boundary of the world-sheet. One might consider all noncyclic permutations of the vertices to get the correct scattering amplitude. However, for our purpose which is comparing string theory S-matrix elements with field theory S-matrix elements, it is really sufficient to consider the S-matrix element with just the factor of $\operatorname{Tr}\left(\lambda^{1} \lambda^{2} \lambda^{3}\right)$. After fixing the $S L(2, R)$, 
one ends up with one double integral which can be performed. Now the integrals are given in terms of the three Mandelstam variables. For the integrals see Appendix B of the paper. Applying these integrals and making use of several identities one can eventually read off the amplitude (17) as the following :

$$
\mathcal{A}^{\phi \phi T C}=\mathcal{A}_{1}+\mathcal{A}_{2}+\mathcal{A}_{3}
$$

where

$$
\begin{aligned}
\mathcal{A}_{1} \sim & 2 i \operatorname{Tr}\left(\lambda_{1} \lambda_{2} \lambda_{3}\right) L_{1}\left\{\left[-\xi_{1 i} \xi_{2 j} k_{1 a} k_{2 b} \operatorname{Tr}\left(P_{-} H_{(n)} M_{p} \Gamma^{j b i a}\right)-p^{i} p^{j} \xi_{1 i} \xi_{2 j} \operatorname{Tr}\left(P_{-} H_{(n)} M_{p}\right)\right.\right. \\
& \left.+\xi_{1 i} \xi_{2 j} p^{i} k_{2 b} \operatorname{Tr}\left(P_{-} H_{(n)} M_{p} \Gamma^{j b}\right)+\xi_{1 i} \xi_{2 j} p^{j} k_{1 a} \operatorname{Tr}\left(P_{-} H_{(n)} M_{p} \Gamma^{i a}\right)\right]\left(-t-s-u-\frac{1}{2}\right) \\
& \left.+\xi_{1} \cdot \xi_{2} \operatorname{Tr}\left(P_{-} H_{(n)} M_{p}\right)\left(\frac{1}{2}\left(u+\frac{1}{4}\right)\left(s+\frac{1}{4}\right)\right)\right\}, \\
\mathcal{A}_{2} \sim & -2 i \operatorname{Tr}\left(\lambda_{1} \lambda_{2} \lambda_{3}\right) L_{2}\left\{2 k_{1 a} k_{2 b} \xi_{1} \cdot \xi_{2} \operatorname{Tr}\left(P_{-} H_{(n)} M_{p} \Gamma^{b a}\right)\right\}, \\
\mathcal{A}_{3} \sim & -2 i \operatorname{Tr}\left(\lambda_{1} \lambda_{2} \lambda_{3}\right) L_{2}\left\{-t \operatorname{Tr}\left(P_{-} H_{(n)} M_{p} \Gamma^{j i}\right) \xi_{1 i} \xi_{2 j}\right\} .
\end{aligned}
$$

The functions $L_{1}, L_{2}$ are :

$$
\begin{aligned}
& L_{1}=(2)^{-2(t+s+u)} \pi \frac{\Gamma\left(-u+\frac{1}{4}\right) \Gamma\left(-s+\frac{1}{4}\right) \Gamma\left(-t+\frac{1}{2}\right) \Gamma\left(-t-s-u-\frac{1}{2}\right)}{\Gamma\left(-u-t+\frac{3}{4}\right) \Gamma\left(-t-s+\frac{3}{4}\right) \Gamma\left(-s-u+\frac{1}{2}\right)}, \\
& L_{2}=(2)^{-2(t+s+u)-1} \pi \frac{\Gamma\left(-u+\frac{3}{4}\right) \Gamma\left(-s+\frac{3}{4}\right) \Gamma(-t) \Gamma(-t-s-u)}{\Gamma\left(-u-t+\frac{3}{4}\right) \Gamma\left(-t-s+\frac{3}{4}\right) \Gamma\left(-s-u+\frac{1}{2}\right)} .
\end{aligned}
$$

Note that all terms including transverse momentum of closed string $p^{i}, p^{j}$ are absent in the amplitude of one RR, two gauge field and one tachyon vertex operators [21]. For their interpretations see section 4 of [8]. Now in order to compare the amplitude of (19) with field theory, we are going to expand (19) to actually produce all infinite number of tachyon and gauge poles. We postpone the expansion to the next section.

Because of the presence $H_{(n)}$ and $\Gamma^{b a}$, one believes that the amplitude is non zero for $p=n-1, p=n-3$ and $p=n+1$ cases. Taking a look at the poles in Gamma functions, one will observe that the amplitude for $p=n+1$ has an infinite number of massless poles and so many contact interactions . 
For the case of $p=n-1$, the amplitude has an infinite number of tachyon poles/contact terms. Whereas, for $p=n-3$ case, there are neither tachyon nor massless poles so the amplitude does include just an infinite number of contact terms. Now one may ask how to expand this amplitude such that the leading terms of the amplitude correspond to the effective actions and non leading terms in our amplitude do belong to the higher derivative terms in the effective actions.

In the next section, concerning momentum conservation along the world volume of brane, sending one Mandelstam variable $(t)$ to zero and sending the other two ones to mass square of tachyon, we conjecture a unique expansion for all four point functions including one RR, massless fields and tachyon. In particular, we will see that massless pole shows that the kinetic term of the scalar field has no higher derivative correction as it has already been set in DBI action.

\section{Remarks on momentum expansion}

The momentum expansion of the amplitude must be achieved by working out in detail either the tachyon or massless pole of field theory. Tachyon action has $U(N)$ gauge symmetry so one might search a unique expansion for all four point functions including one RR, massless fields and tachyon. To discover this expansion, we must remember the fact that transverse scalar field and tachyon of $\mathrm{D}_{8}$-brane transform in the adjoint representation of $U(N)$ group. Therefore one may conclude that both of them should have the same non-abelian kinetic term, as it is the case. Note that their Feynman diagrams (taking their kinetic term into account) are also equal.

Recalling the relation between Mandelstam variables and the momentum of RR field (11), one does believe this on-shell constraint does not permit us to send all $s, t, u$ to zero. Once more note that all on-shell conditions imply that the RR field must be non-zero, i.e., $p_{a} p^{a} \rightarrow 1 / 4$. Thus we come to the point that computations make sense just for the non-BPS euclidean SD-brane. Hence, once more due to some kinematic reason [23, 56] the S-matrix method must be used to confirm all Wess-Zumino couplings only in the presence of nonBPS SD-branes [36]. It is worth to talking about on-shell condition for RR 's momentum as follows:

$$
p_{i} p^{i}+p_{a} p^{a}=0
$$


For the definition of $\mathrm{SD}_{p}$-brane see [22. Having used the kinematic relation (11), in which the Mandelstam variables are satisfied, one can precisely reproduce all leading terms of the expansion with making use of the tachyon action. Concerning the fact that these relations involve the mass of tachyon, one really believes that the tachyon potential should include tachyon mass as well.

There are two different assumptions in order to get the correct momentum expansion of a S-matrix element which are either $\left(k_{i}+k_{j}\right)^{2} \rightarrow 0$ or $k_{i} \cdot k_{j} \rightarrow 0$. The case $\left(k_{i}+k_{j}\right)^{2} \rightarrow 0$ so happens once we encounter a simple massless pole in $\left(k_{i}+k_{j}\right)^{2}$-channel. Applying this fact, we understand that our amplitude $(C \phi \phi T)$ just has a simple massless pole in $\left(k_{1}+k_{2}\right)^{2}$ channel. Thus the correct momentum expansion in accord with the above argument must be done around

$$
k_{3} \cdot k_{1} \rightarrow 0, \quad k_{3} \cdot k_{2} \rightarrow 0, \quad\left(k_{1}+k_{2}\right)^{2} \rightarrow 0
$$

Now we may use on-shell relations $k_{1}^{2}=k_{2}^{2}=0$ and $k_{3}^{2}=1 / 4$, to rewrite (21) as

$$
s \rightarrow-1 / 4, \quad u \rightarrow-1 / 4, \quad t \rightarrow 0,
$$

Therefore as it is clear, because of tachyon pole, the expansion is not usual $\alpha^{\prime}$ expansion any more. So tachyon and massive modes will be decoupled. Our main goal is to find out new couplings for which precisely reproduce all terms of the expansion.

Regarding above remarks, the expansion of the function $L_{1}$ around (22) is

$$
\begin{aligned}
L_{1}= & -\pi^{5 / 2}\left(\sum_{n=0}^{\infty} c_{n}\left(s^{\prime}+t+u^{\prime}\right)^{n}+\frac{\sum_{n, m=0}^{\infty} c_{n, m}\left[\left(s^{\prime}\right)^{n}\left(u^{\prime}\right)^{m}+\left(s^{\prime}\right)^{m}\left(u^{\prime}\right)^{n}\right]}{\left(t+s^{\prime}+u^{\prime}\right)}\right. \\
& \left.+\sum_{p, n, m=0}^{\infty} f_{p, n, m}\left(s^{\prime}+t+u^{\prime}\right)^{p}\left[\left(s^{\prime}+u^{\prime}\right)^{n}\left(s^{\prime} u^{\prime}\right)^{m}\right]\right),
\end{aligned}
$$

Note that the structure of contact interaction terms in (23) is not the same. Hence, we believe contact terms in the second line of (23) are related to different couplings as we will derive them in the field theory side as well. They were corresponding to different field theory couplings. The expansion of the function $L_{2}$ around (22) is

$$
L_{2}=-\pi^{3 / 2}\left(\frac{1}{t} \sum_{n=-1}^{\infty} b_{n}\left(u^{\prime}+s^{\prime}\right)^{n+1}+\sum_{p, n, m=0}^{\infty} e_{p, n, m} t^{p}\left(s^{\prime} u^{\prime}\right)^{n}\left(s^{\prime}+u^{\prime}\right)^{m}\right) .
$$

where in the above relations we have considered $u^{\prime}=u+\frac{1}{4}=-\alpha^{\prime} k_{2} \cdot k_{3}$ and $s^{\prime}=s+\frac{1}{4}=$ $-\alpha^{\prime} k_{1} \cdot k_{3}$. One important point that should be made is that all $b_{n}$ coefficients are the same 
as those appeared in the momentum expansion of the amplitude of one RR, three massless open string vertex operators for BPS branes [6, 7, 8]. Let us write some of them down

$$
\begin{aligned}
& b_{-1}=1, b_{0}=0, b_{1}=\frac{1}{6} \pi^{2}, b_{2}=2 \zeta(3), \\
& e_{2,0,0}=e_{0,1,0}=2 \zeta(3), e_{1,0,0}=\frac{1}{6} \pi^{2}, e_{1,0,2}=\frac{19}{60} \pi^{4}, e_{1,0,1}=e_{0,0,2}=6 \zeta(3), \\
& e_{0,0,1}=\frac{1}{3} \pi^{2}, e_{3,0,0}=\frac{19}{360} \pi^{4}, e_{0,0,3}=e_{2,0,1}=\frac{19}{90} \pi^{4}, e_{1,1,0}=e_{0,1,1}=\frac{1}{30} \pi^{4}, \\
& c_{0}=0, c_{1}=\frac{\pi^{2}}{3}, c_{2}=4 \zeta(3), c_{1,1}=\frac{\pi^{2}}{3}, c_{0,0}=1, c_{3,1}=c_{1,3}=\frac{4 \pi^{4}}{15}, c_{2,2}=\frac{2 \pi^{4}}{15} \\
& c_{1,0}=c_{0,1}=0, c_{3,0}=c_{0,3}=0 c_{2,0}=c_{0,2}=\frac{\pi^{2}}{3}, c_{1,2}=c_{2,1}=-8 \zeta(3), \\
& f_{0,1,0}=-\frac{2 \pi^{2}}{3}, f_{0,2,0}=-f_{1,1,0}=12 \zeta(3), f_{0,0,1}=4 \zeta(3) c_{4,0}=c_{0,4}=\frac{2 \pi^{4}}{15}
\end{aligned}
$$

However, some of the coefficients, in particular $c_{n, m}$ are different from those that appeared in [6] because the expansions are not the same. For the amplitude of $C A A A$ the expansion was low energy expansion while here the expansion is not low energy expansion even though apparently they do have the same structure. We will see that these coefficients play a key role in confirming the infinite number of tachyon poles in string theory. Now it becomes clear that $L_{1}$ has an infinite number of tachyon poles in $s^{\prime}+t+u^{\prime}$-channel and $L_{2}$ has an infinite number of massless poles in $t$-channel. These poles must be reproduced in field theory by well defined couplings. In the next section, we talk about effective field theory, then we try to produce the first simple tachyon pole within field theory context, then we go on to find all of the infinite numbers of the couplings between two scalar fields and two tachyons to all orders of $\alpha^{\prime}$, which can be approved just by S-matrix computations.

\section{Effective Field Theory on the World-Volume}

On-shell states in our amplitude are in fact open string tachyon and scalar fields which are coming from DBI action and the closed string RR field which is coming from the WessZumino action. One may wonder whether there could be an off-shell gauge field in our amplitude. We confirm its existence in the field theory side. We are also working in flat background. Applying square root expansion mentioned in [6] we could find non abelian kinetic terms as

$$
\mathcal{L}=-T_{p}\left(\pi \alpha^{\prime}\right) \operatorname{Tr}\left(m^{2} T^{2}+D_{a} T D^{a} T-\left(\pi \alpha^{\prime}\right) F_{a b} F^{b a}\right)
$$


In order to actually have gauge field and tachyon propagators we need to keep them as well.

On the other hand, the scalar fields have geometrical meaning which represent transverse coordinates of the brane in effective theory.

One has to mention that scalars have $U(N)$ gauge symmetry and they have to be in the adjoint representation. In general scalars do appear in the effective action in three different ways and we now list them .

In the first way, they have been confirmed in Wess-Zumino action in its exponential (3). So our notation is as follows

$$
i_{\Phi} i_{\Phi} C^{(n)}=\frac{1}{2(n-2) !}\left[\Phi^{i}, \Phi^{j}\right] C_{j i \mu_{3} \cdots \mu_{n}}^{(n)} d x^{\mu_{3}} \cdots d x^{\mu_{n}}, \quad C^{(n)}=\frac{1}{n !} C_{\mu_{1} \cdots \mu_{n}}^{(n)} d x^{\mu_{1}} \cdots d x^{\mu_{n}}
$$

In the second way, we will have the covariant derivatives of the non abelian scalar fields in pullback as follows

$$
P[E]_{a b}=E_{a b}+\lambda E_{a i} D_{b} \Phi^{i}+\lambda E_{i b} D_{a} \Phi^{i}+\lambda^{2} E_{i j} D_{a} \Phi^{i} D_{b} \Phi^{j},
$$

In the last way,the metric will be given by a non-abelian Taylor expansion as

$$
\begin{aligned}
G_{\mu \nu} & =\left.\exp \left[\lambda \Phi^{i} \partial_{x^{i}}\right] G_{\mu \nu}^{0}\left(\sigma^{a}, x^{i}\right)\right|_{x^{i}=0} \\
& =\left.\sum_{n=0}^{\infty} \frac{\lambda^{n}}{n !} \Phi^{i_{1}} \cdots \Phi^{i_{n}}\left(\partial_{x^{i_{1}}} \cdots \partial_{x^{i_{n}}}\right) G_{\mu \nu}^{0}\left(\sigma^{a}, x^{i}\right)\right|_{x^{i}=0} .
\end{aligned}
$$

Hence, one might reveal that the action does involve all transverse derivatives of the closed string RR fields within Taylor expansion.

The existence of the scalar fields in pullback [57] and the functional dependence [58] have already been addressed. Here we would like to establish the explicit existence of the commutator interactions of scalar fields even to tachyon in Wess-Zumino action. Another ambitious goal is to obtain some sort of new couplings which will be investigated by honest string scattering computations which will be explained in the section of contact terms. In order to actually have some non trivial couplings, we have to have at least three kinds of open string states and a single closed string state. One may point out that the leading terms for scalar fields could have been found from Born-Infeld action as

$$
-\lambda^{2} T_{p} \int d^{p+1} \sigma \operatorname{Tr}\left(\frac{1}{2} D^{a} \Phi^{i} D_{a} \Phi^{i}-\frac{1}{4}\left[\Phi^{i}, \Phi^{j}\right]\left[\Phi^{i}, \Phi^{j}\right]\right)
$$


One more thing which is really worth saying is that pullback of closed string fields must be defined in the static gauge. Also notice that in the pullback we must have covariant derivatives of non abelian scalars as

$$
P\left[\eta_{a b}\right]=\eta_{a b}+2 \pi \alpha^{\prime} D_{a} \phi^{i} D_{b} \phi^{j} \eta_{i j}
$$

The first term in (30) is indeed non abelian kinetic term of scalar fields which can be regarded as a reduction of $F^{2}$ in ten dimensions.

Within our conventions, $D_{a} \Phi^{i}=\partial_{a} \Phi^{i}+i\left[A_{a}, \Phi^{i}\right]$. Note that the non abelian scalar field theory now does involve the interactions with even gauge fields, which are either inside of three-point function $\operatorname{Tr}\left(\partial^{a} \Phi^{i}\left[A_{a}, \Phi^{i}\right]\right)$ or four-point function $\operatorname{Tr}\left(\left[A^{a}, \Phi_{i}\right]\left[A_{a}, \Phi^{i}\right]\right)$. So we expect from the field theory point of view, Feynman diagrams in which two open scalars may scatter to give an off-shell gauge field which is going to be absorbed by a lower order RR coupling (in the bulk) and one external tachyon in the world volume space. This does happen for $p=n+1$ case. Thus we will see in field theory this diagram is responsible for an infinite number of massless gauge poles indicating in the string amplitude. We also determine all their contact terms .

Notice the fact that due to the appearance of unusual kinematics and the mass of tachyon we are not able to derive our amplitude from an usual five-point open string amplitude. That is why we have done the computations of this amplitude directly, which are indeed more sophisticated than five-point pure open string amplitude .

\section{$5.1 p=n-1$ case}

Having set the usual Chern-Simons action, it seems that there is no coupling between RR fields of the type II string theory and the non-BPS D-branes. However, one must point out that there is a nonvanishing coupling between the RR field and one tachyon on the world-volume of these branes, such that the Chern-Simons action was modified in [56] to be responsible for this coupling.

In this section we would like to obtain the first tachyonic pole of the amplitude for

$p=n-1$ case and then we proceed to discover an infinite number of higher derivative corrections that are related to two tachyons and two scalar fields. In order to show that our proposal for these infinite numbers of couplings works we use these couplings to check the infinite number of tachyon poles of the amplitude for $p=n-1$ case later on. Finally we will consider all contact terms together. Only the last term in $\mathcal{A}_{1}$ in (19) is related to 
singular terms. Therefore for this case the trace is:

$$
\operatorname{Tr}\left(H_{(n)} M_{p}\right)= \pm \frac{32}{n !} \epsilon^{a_{0} \cdots a_{p}} H_{a_{0} \cdots a_{p}}
$$

Note that the trace also does involve the factor of $\gamma^{11}$ and keeps held the following results for $p>3$ with $H_{(n)} \equiv * H_{(10-n)}$ for $n \geq 5$. Replacing this trace in the last term of $\mathcal{A}_{1}$, one gets

$$
\mathcal{A}^{\phi \phi T C}=\frac{\mp 32 i}{(p+1) !}\left(\beta^{\prime} \mu_{p}^{\prime} \pi^{1 / 2}\right) \operatorname{Tr}\left(\lambda_{1} \lambda_{2} \lambda_{3}\right) \epsilon^{a_{0} \cdots a_{p}} H_{a_{0} \cdots a_{p}} L_{1}\left\{\frac{1}{2}\left(\xi_{1} \cdot \xi_{2}\right)\left(u+\frac{1}{4}\right)\left(s+\frac{1}{4}\right)\right\}
$$

where $\left(\beta^{\prime} \mu_{p}^{\prime} \pi^{1 / 2}\right)$ is a normalization factor. As it is clear (31) is symmetric under changing massless scalar fields, therefore the amplitude is non-zero even for the abelian gauge group. Notice that we do not want to fix the sign of the amplitudes.

It is seen that (31) has an infinite number of tachyon poles in the $\left(s^{\prime}+t+u^{\prime}\right)$-channel and does include an infinite number of contact terms. In the next section we would like to show that just the first tachyon pole in (31) will be reproduced by applying the symmetric trace prescription of tachyonic DBI action. However, in order to be able to produce an infinite number of tachyon poles of the desired amplitude up to all orders of $\alpha^{\prime}$, one must find an infinite number of higher derivative corrections of two scalar fields and two tachyons. Making use of their explicit forms and extracting the vertex of two on-shell scalars, one tachyon and one off-shell tachyon in the world-volume theory of $\mathrm{N}$ coincident non-BPS branes, we will be able to produce all infinite number of tachyon poles in (31) in a precise manner.

\subsection{First Tachyon pole for $p=n-1$ case}

Here we are going to mention the general form of tachyonic action [25, 23] as the following:

$$
\begin{aligned}
S_{D B I}=-\frac{T_{p}}{2} \int d^{p+1} & \sigma \operatorname{STr}\left(V\left(T_{i} T_{i}\right) \sqrt{1+\frac{1}{2}\left[T_{i}, T_{j}\right]\left[T_{j}, T_{i}\right]}\right. \\
\times & \left.\sqrt{-\operatorname{det}\left(\eta_{a b}+2 \pi \alpha^{\prime} F_{a b}+2 \pi \alpha^{\prime} D_{a} T_{i}\left(Q_{1}^{-1}\right)_{i j} D_{b} T_{j}\right)}\right),
\end{aligned}
$$

where the tachyon potential appeared in (9) and

$$
Q_{1 i j}=I \delta_{i j}-i\left[T_{i}, T_{j}\right]
$$


We must take into account the fact that the trace in the action is totally symmetric between $F_{a b}, D_{a} T^{i},\left[T^{i}, T^{j}\right]$ and $T^{i}$ inside the potential $V\left(T^{i} T^{i}\right)$.

One can write down $V(T)$ around its maximum which is $T_{\max }=0$ so that

$$
V(T)=1-\frac{\pi}{2} T^{2}+\frac{\pi^{2}}{8} T^{4}+O\left(T^{6}\right)
$$

As observed with A.Sen 's conjecture the coefficient of $T^{4}$ is a consistent result which indicates that the tachyon potential has a minimum [44, 59]. Therefore one can see that by neglecting $O\left(T^{6}\right)$ terms, $V(T)$ does have a minimum at $T_{\min }=\sqrt{2 / \pi}$, where the value of the potential at its minimum is $V\left(T_{\min }\right)=0.5$.

So we conclude that $V\left(T_{\min }\right)$ is now non zero; however, at real stable vacuum the value of the potential must be zero so $V\left(T_{\min }\right)=0.5$ means that one must consider the higher order $O\left(T^{6}\right)$ terms in the potential. The other point we want to make is that, A.Sen showed that the minimum of the potential is at $T_{\min } \rightarrow \infty$, and $V(T)$ has exponential behaviour just like $e^{-\sqrt{\pi} T}$ around its minimum [40].

However within our formalism we want to study this action around the unstable point $T=0$ and around $T \rightarrow \infty$ just the second term in (34) is dominant. Thus, above action does reduce to usual action with potential $T^{4} V\left(T^{2}\right)$ and at $T \rightarrow \infty$ this potential tends to zero. This is already expected from tachyon condensation for a single non-BPS brane. To check the consistency (32) with some disk level amplitudes in super string theory see [21, 25].

Applying the expansion of the square root of determinant $\sqrt{\operatorname{det}\left(M_{0}+M\right)}[6]$ and making use of both actions (11) and (32), one gets various interactions. However, we are interested in considering two scalar and two tachyon couplings thus we will have the following couplings:

$$
\begin{aligned}
\mathcal{L}(\phi, \phi, T, T)= & -2 T_{p}\left(\pi \alpha^{\prime}\right)^{3} \operatorname{STr}\left(m^{2} T^{2}\left(D_{a} \phi^{i} D^{a} \phi_{i}\right)+\frac{\alpha^{\prime}}{2} D^{\alpha} T D_{\alpha} T D_{a} \phi^{i} D^{a} \phi_{i}\right. \\
& \left.-\alpha^{\prime} D^{b} T D^{a} T D_{a} \phi^{i} D_{b} \phi_{i}\right) .
\end{aligned}
$$

Note that in the above couplings one tachyon has to be off-shell and the external states are two scalars and one tachyon in which they must carry their own momentum. That is why the coupling $\frac{1}{4 \pi \alpha^{\prime}}\left[X^{i}, T\right]\left[X_{i}, T\right]$ has been over looked. One more point to mention is that after averaging all possible permutations in (35) one has to take also an overall trace on the group theory. We want to obtain the higher derivative couplings of the two scalars 
and two tachyons and then investigate that these terms reproduce an infinite number of tachyon poles in the amplitude, and finally we end up with some new couplings which can be confirmed just by direct scattering amplitude computations.

Because of the fact that propagator is Abelian, we need to consider two possible orderings in order to get $\operatorname{Tr}\left(\lambda_{1} \lambda_{2} \lambda_{3}\right)$ ordering. Having written down symmetric traces in terms of ordinary traces, one can essentially find out the terms $\left(L_{1}^{0,0}+L_{2}^{0,0}+L_{3}^{0,0}+L_{4}^{0,0}\right)$ such that

$$
\mathcal{L}=-2 T_{p}\left(\pi \alpha^{\prime}\right)^{3}\left(\mathcal{L}_{1}^{0,0}+\mathcal{L}_{2}^{0,0}+\mathcal{L}_{3}^{0,0}+\mathcal{L}_{4}^{0,0}\right)
$$

where

$$
\begin{aligned}
\mathcal{L}_{1}^{0,0} & =\frac{-4 m^{2}}{\pi^{2}} \operatorname{Tr}\left(a_{0,0}\left(T^{2} D_{a} \phi^{i} D^{a} \phi_{i}\right)+b_{0,0}\left(T D_{a} \phi^{i} T D^{a} \phi_{i}\right)\right) \\
\mathcal{L}_{2}^{n m} & =\frac{-4}{\pi^{2}} \operatorname{Tr}\left(a_{0,0}\left(D^{\alpha} T D_{\alpha} T D_{a} \phi^{i} D^{a} \phi_{i}\right)+b_{0,0}\left(D^{\alpha} T D_{a} \phi^{i} D_{\alpha} T D^{a} \phi_{i}\right)\right), \\
\mathcal{L}_{3}^{n m} & =\frac{4}{\pi^{2}} \operatorname{Tr}\left(a_{0,0}\left(D^{\beta} T D_{\mu} T D^{\mu} \phi^{i} D_{\beta} \phi_{i}\right)+b_{0,0}\left(D^{\beta} T D^{\mu} \phi^{i} D_{\mu} T D_{\beta} \phi_{i}\right)\right), \\
\mathcal{L}_{4}^{n m} & =\frac{4}{\pi^{2}} \operatorname{Tr}\left(a_{0,0}\left(D^{\beta} T D^{\mu} T D_{\beta} \phi^{i} D_{\mu} \phi_{i}\right)+b_{0,0}\left(D^{\beta} T D_{\beta} \phi^{i} D^{\mu} T D_{\mu} \phi_{i}\right)\right) .
\end{aligned}
$$

with $a_{0,0}=\frac{-\pi^{2}}{6}, b_{0,0}=\frac{-\pi^{2}}{12}$. Let us mention the useful relation $\mathrm{S} \operatorname{Tr}\left(T^{2} L L\right)=\frac{2}{3} \operatorname{Tr}(T T L L)+$ $\frac{1}{3} \operatorname{Tr}(T L T L)$. Thus the first tachyon pole of the amplitude (31) may be found as

$$
\mathcal{A}=V_{\alpha}\left(C_{p}, T\right) G_{\alpha \beta}(T) V_{\beta}\left(T, T_{3}, \phi_{1}, \phi_{2}\right),
$$

The tachyon propagator and the vertex $V_{\alpha}\left(C_{p}, T\right)$ which have no higher derivative correction might be appeared as

$$
\begin{aligned}
G^{\alpha \beta}(T) & =\frac{i \delta^{\alpha \beta}}{\left(2 \pi \alpha^{\prime}\right) T_{p}\left(-k^{2}-m^{2}\right)} \\
V^{\alpha}\left(C_{p}, T\right) & =2 i \mu_{p}^{\prime} \beta^{\prime}\left(2 \pi \alpha^{\prime}\right) \frac{1}{(p+1) !} \epsilon^{a_{0} \cdots a_{p}} H_{a_{0} \cdots a_{p}} \operatorname{Tr}\left(\Lambda^{\alpha}\right) .
\end{aligned}
$$

$\alpha, \beta$ are group indices. Note that in (39), $\operatorname{Tr}\left(\Lambda^{\alpha}\right)$ is nonzero just for Abelian matrix $\Lambda^{\alpha}$. The vertex $V^{\beta}\left(T, T_{3}, \phi_{1}, \phi_{2}\right)$ can be derived from (36). Recalling that off-shell tachyon must be Abelian, one gets

$$
V^{\beta}\left(T, T_{3}, \phi_{1}, \phi_{2}\right)=4 i T_{p}\left(\pi^{3} \alpha^{\prime}\right)\left(\alpha^{\prime}\right)^{2} \operatorname{Tr}\left(\lambda_{1} \lambda_{2} \lambda_{3} \Lambda^{\beta}\right)\left[\frac{1}{2}\left(\xi_{1} \cdot \xi_{2}\right)\left(u+\frac{1}{4}\right)\left(s+\frac{1}{4}\right)\right],
$$

Note that in order to obtain this vertex we have to consider all two group factors of $\operatorname{Tr}\left(\lambda_{3} \Lambda^{\beta} \lambda_{1} \lambda_{2}\right)$ and $\operatorname{Tr}\left(\Lambda^{\beta} \lambda_{3} \lambda_{1} \lambda_{2}\right)$. 
However, the other terms will have the contribution of $\operatorname{Tr}\left(\lambda_{2} \lambda_{1} \lambda_{3} \Lambda^{\beta}\right)$ to (40). Having replaced (40) in the Feynman amplitude (38), we get the following result in the field theory side:

$$
-64 i \pi^{3} \beta^{\prime} \mu_{p}^{\prime} \frac{\epsilon^{a_{0} \cdots a_{p}} H_{a_{0} \cdots a_{p}}}{(p+1) !\left(s^{\prime}+t+u^{\prime}\right)} \operatorname{Tr}\left(\lambda_{1} \lambda_{2} \lambda_{3}\right)\left[\left(\xi_{1} \cdot \xi_{2}\right) \frac{1}{2} u^{\prime} s^{\prime}\right] .
$$

Notice that we just considered the first tachyon pole of $L_{1}$, which had the coefficient of $2 \pi^{\frac{5}{2}}$. Thus symmetrized trace prescription of tachyonic DBI action could precisely reproduce the first tachyon pole of string theory amplitude. How can we produce the other tachyon poles? In detail below we obtain all couplings between two tachyons and two scalars to all orders in $\alpha^{\prime}$ and then use them we produce all infinite numbers of tachyon poles for $p=n-1$ case in the field theory side as well.

\section{Two scalar and two tachyon couplings up to all or- ders of $\alpha^{\prime}$}

It is known the higher derivative corrections do have some kind of field redefinition freedom. Therefore we may be able to pick this freedom up and relate it to some couplings in field theory. For example we can relate $\left(\alpha^{\prime}\right)^{3}$ terms to the couplings that involve $\partial \partial \partial T$ and so on.

It is indeed an interesting issue to find out these higher derivative terms for all orders of $\alpha^{\prime}$ which is one of the main goals of the paper.

A precise method for obtaining the general form of higher derivative theories is indeed studying in detail the S-matrix method. Using this method one first has to find S-matrix elements of the desired theory and then try to compare them with the S-matrix elements of super string theory. If these higher derivative terms are equal with the string theory, then their S-matrix elements must be the same with the momentum expansion of the Smatrix elements of string theory. Therefore, in order to get those couplings, we should look for S-matrix elements and try to expand them in such a way that the correct higher derivative couplings are discovered in field theory. As an example, in order to get the Smatrix elements of two real tachyons and one closed string RR field, the following higher derivative couplings in brane-antibrane must be taken into account:

$$
2 i \alpha^{\prime} \mu_{p} \sum_{n=0}^{\infty} a_{n}\left(\frac{\alpha^{\prime}}{2}\right)^{n} C_{p-1} \wedge\left(D^{a} D_{a}\right)^{n}\left(D T \wedge D T^{*}\right) .
$$


For more information see [21, 60].

However, for four and five-point functions, it is really a complicated task to find higher derivative corrections. Let us address an issue. The S-matrix elements of tachyons might not have definite physical meaning; nevertheless, if the two theories are equal then we believe that the string theory S-matrix elements must be reproduced just by their higher derivative couplings.

One important point on tachyonic action is that it produces just leading terms of Smatrix elements at the top of tachyon potential (not $\alpha^{\prime} \rightarrow 0$ limit). Therefore we may expect already that the other terms of expansion really have important effect. So one concludes that in field theory effective action must have all those higher derivative terms as well. Let us come to our main point.

In this section we would like to obtain the infinite number of the couplings between two scalars and two tachyons in order to reproduce the string theory S-matrix elements to all orders of $\alpha^{\prime}$.

Here we just mention our method for finding the higher derivative extensions of the above couplings (37). Two important points are worth mentioning. The first point is that the kinematic factor in the amplitude of two tachyons and two scalar fields, is the vertex of two on-shell tachyons and two on-shell scalar fields of (37) and the second point is that, the coefficient of all higher order terms in the amplitude of two tachyons and two scalar fields is exactly the vertex of two tachyons and two scalars. Thus we might find out the higher derivative couplings by acting suitable higher derivatives on these couplings (37). Each term in the above couplings has the coefficient $a_{0,0}$ and $b_{0,0}$. In the higher derivative orders one must substitute them by $a_{n, m}$ and $b_{n, m}$. For further details see [6, 7, 8, 23, 60].

Concerning T-duality transformation, one may expect that the higher derivative couplings of two scalar fields and two tachyons might be similar to the higher derivative couplings of two gauge fields and two tachyons. To realize the differences for gauge and scalar field see section 5 of [6].

Because of two important facts one may try to apply the general form of T-duality transformation to discover higher derivative couplings two scalar fields and two tachyons on the world volume of $N$ non-BPS D-branes, to all orders of $\alpha^{\prime}$ and then precisely fix their coefficients comparing with S-matrix elements.

Let us make some comments. The first comment is that the Mandelstam variables 
for the amplitudes of TT $\phi \phi$ and TTAA must satisfy the same constraint. Notice that all massless poles coming from the non-Abelian kinetic term have to be reproduced by sending some of the Mandelstam variables to zero. The second point that should be made is that, all external states in those two amplitudes satisfy the same on-shell and physical state condition .

The method for finding those infinite numbers of couplings between two gauge fields and two tachyons has been mentioned in [23, 60].

The massless poles in the amplitude of TTAA have been reproduced by the non-Abelian kinetic term of the gauge fields and their Lagrangian is

$$
\mathcal{L}(A, A, T, T)=\left(\pi \alpha^{\prime}\right)^{3} \mathrm{~S} \operatorname{Tr}\left(m^{2} T^{2} F_{\mu \nu} F^{\nu \mu}+D^{\alpha} T D_{\alpha} T F_{\mu \nu} F^{\nu \mu}-4 F^{\mu \alpha} F_{\alpha \beta} D^{\beta} T D_{\mu} T\right) .
$$

However, for the amplitude of $T T \phi \phi$ the massless poles are reproduced by the non-Abelian kinetic term of the scalar fields and their Lagrangian appears in (35).

If we compare the above Lagrangian with (35) then we realize that they have some differences in the the indices and coefficients. In order to replace $F$ with $D \phi$ and to observe more details see section 5 of [6].

To avoid further details we just write down the results that we found using direct SMatrix computations.

Therefore by extracting symmetrized traces in terms of usual traces we were able to find out the couplings between two tachyons and two covariant derivative of scalar fields on the world volume of $N$ coincident non-BPS D-branes, to all orders of $\alpha^{\prime}$ as the following:

$$
\mathcal{L}=-2 T_{p}\left(\pi \alpha^{\prime}\right)\left(\alpha^{\prime}\right)^{2+n+m} \sum_{n, m=0}^{\infty}\left(\mathcal{L}_{1}^{n m}+\mathcal{L}_{2}^{n m}+\mathcal{L}_{3}^{n m}+\mathcal{L}_{4}^{n m}\right)
$$

where

$$
\begin{aligned}
\mathcal{L}_{1}^{n m}= & m^{2} \operatorname{Tr}\left(a_{n, m}\left[\mathcal{D}_{n m}\left(T^{2} D_{a} \phi^{i} D^{a} \phi_{i}\right)+\mathcal{D}_{n m}\left(D_{a} \phi^{i} D^{a} \phi_{i} T^{2}\right)\right]\right. \\
& \left.+b_{n, m}\left[\mathcal{D}_{n m}^{\prime}\left(T D_{a} \phi^{i} T D^{a} \phi_{i}\right)+\mathcal{D}_{n m}^{\prime}\left(D_{a} \phi^{i} T D^{a} \phi_{i} T\right)\right]+h . c .\right), \\
\mathcal{L}_{2}^{n m}= & \operatorname{Tr}\left(a_{n, m}\left[\mathcal{D}_{n m}\left(D^{\alpha} T D_{\alpha} T D_{a} \phi^{i} D^{a} \phi_{i}\right)+\mathcal{D}_{n m}\left(D_{a} \phi^{i} D^{a} \phi_{i} D^{\alpha} T D_{\alpha} T\right)\right]\right. \\
& \left.+b_{n, m}\left[\mathcal{D}_{n m}^{\prime}\left(D^{\alpha} T D_{a} \phi^{i} D_{\alpha} T D^{a} \phi_{i}\right)+\mathcal{D}_{n m}^{\prime}\left(D_{a} \phi^{i} D_{\alpha} T D^{a} \phi_{i} D^{\alpha} T\right)\right]+\text { h.c. }\right),
\end{aligned}
$$




$$
\begin{aligned}
\mathcal{L}_{3}^{n m}= & -\operatorname{Tr}\left(a_{n, m}\left[\mathcal{D}_{n m}\left(D^{\beta} T D_{\mu} T D^{\mu} \phi^{i} D_{\beta} \phi_{i}\right)+\mathcal{D}_{n m}\left(D^{\mu} \phi^{i} D_{\beta} \phi_{i} D^{\beta} T D_{\mu} T\right)\right]\right. \\
& \left.+b_{n, m}\left[\mathcal{D}_{n m}^{\prime}\left(D^{\beta} T D^{\mu} \phi^{i} D_{\mu} T D_{\beta} \phi_{i}\right)+\mathcal{D}_{n m}^{\prime}\left(D^{\mu} \phi^{i} D_{\mu} T D_{\beta} \phi_{i} D^{\beta} T\right)\right]+\text { h.c. }\right), \\
\mathcal{L}_{4}^{n m}= & -\operatorname{Tr}\left(a_{n, m}\left[\mathcal{D}_{n m}\left(D^{\beta} T D^{\mu} T D_{\beta} \phi^{i} D_{\mu} \phi_{i}\right)+\mathcal{D}_{n m}\left(D^{\beta} \phi^{i} D^{\mu} \phi_{i} D_{\beta} T D_{\mu} T\right)\right]\right. \\
& \left.+b_{n, m}\left[\mathcal{D}_{n m}^{\prime}\left(D^{\beta} T D_{\beta} \phi^{i} D^{\mu} T D_{\mu} \phi_{i}\right)+\mathcal{D}_{n m}^{\prime}\left(D_{\beta} \phi^{i} D_{\mu} T D^{\mu} \phi_{i} D^{\beta} T\right)\right]+\text { h.c. }\right),
\end{aligned}
$$

If we did calculate the coupling of two on-shell tachyons and two on-shell scalar fields from (43), one would be able to talk about all the interactions in the amplitude of two scalar fields and two tachyons. The higher derivative operator $\mathcal{D}_{n m}$ and $\mathcal{D}_{n m}^{\prime}$ may be read as

$$
\begin{aligned}
& \mathcal{D}_{n m}(E F G H) \equiv D_{b_{1}} \cdots D_{b_{m}} D_{a_{1}} \cdots D_{a_{n}} E F D^{a_{1}} \cdots D^{a_{n}} G D^{b_{1}} \cdots D^{b_{m}} H \\
& \mathcal{D}_{n m}^{\prime}(E F G H) \equiv D_{b_{1}} \cdots D_{b_{m}} D_{a_{1}} \cdots D_{a_{n}} E D^{a_{1}} \cdots D^{a_{n}} F G D^{b_{1}} \cdots D^{b_{m}} H
\end{aligned}
$$

So (43) is the higher derivative correction of two scalar field and two tachyon couplings of tachyonic action. One important evidence in confirming (43) is that by setting the covariant derivative of the scalar field and the second covariant derivative of tachyon to zero, (43) goes back to the couplings (35). This definitely shows that when fields vary so slowly, the non-Abelian tachyon DBI action is going to be the correct effective action for non-BPS SD-branes.

One extremely important fact about the couplings in (43) is that they may have on-shell ambiguity, which means that there is no difference between $T$ and $2 \alpha^{\prime} D_{a} D^{a} T$ because they do have an identical effect. However, this ambiguity does not have any effect on the simple massless and tachyon poles of the amplitude. In the case of massless poles it is sort of an obvious thing because the tachyons are on-shell. In the case of tachyon poles we will show that $k^{2}+m^{2}$ terms cancelled by tachyon pole, and eventually one gets some contact terms. Thus the difference is just an extra contact interaction. By doing an amplitude where the couplings (43) would appear either in tachyon poles or contact terms, one will be able to fix that ambiguity in (43). These couplings might appear in the tachyonic pole of S-matrix elements of two tachyons, two scalar fields and one gauge field. It would be nice to follow in detail this amplitude, in favor of those couplings. This amplitude does have a long computation that we leave for future work [61].

In the next section we will show that an infinite number of the tachyon poles will 
result by taking the Wess-Zumino coupling $C_{p} \wedge D T$ and by our proposed higher derivative couplings of two scalar fields and two tachyons, which we discovered in (43).

\subsection{Infinite number of tachyon poles for $p+1=n$ case}

As we promised in this section we are going to investigate that the infinite number of two scalar field and two tachyon couplings in (43) will result in the infinite number of tachyon poles of the string theory amplitude in the $\left(s^{\prime}+t+u^{\prime}\right)$-channel. To do so, we must take the following Feynman diagram where one RR in the world volume of non-BPS branes decays to one tachyon and two scalar fields as follows :

$$
\mathcal{A}=V^{\alpha}\left(C_{p}, T\right) G^{\alpha \beta}(T) V^{\beta}\left(T, T_{3}, \phi_{1}, \phi_{2}\right)
$$

In order to proceed one needs the vertex of one RR p-form field and one off-shell tachyon and tachyon propagator as

$$
\begin{aligned}
G^{\alpha \beta}(T) & =\frac{i \delta^{\alpha \beta}}{\left(2 \pi \alpha^{\prime}\right) T_{p}\left(-k^{2}-m^{2}\right)} \\
V^{\alpha}\left(C_{p}, T\right) & =2 i \mu_{p}^{\prime} \beta^{\prime}\left(2 \pi \alpha^{\prime}\right) \frac{1}{(p+1) !} \epsilon^{a_{0} \cdots a_{p}} H_{a_{0} \cdots a_{p}} \operatorname{Tr}\left(\Lambda^{\alpha}\right) .
\end{aligned}
$$

Note that $\operatorname{Tr}\left(\Lambda^{\alpha}\right)$ makes sense just for the Abelian gauge group. One also needs to find out the vertex of one off-shell,one on-shell tachyon and two on-shell scalar fields. This vertex must be obtained from (43). Regarding off-shell tachyon as the Abelian one, we get the vertex of $V^{\beta}\left(T, T_{3}, \phi_{1}, \phi_{2}\right)$ as follows

$$
\begin{aligned}
& 2 i T_{p}\left(\pi \alpha^{\prime}\right)\left(\alpha^{\prime}\right)^{2+n+m}\left(a_{n, m}+b_{n, m}\right) \operatorname{Tr}\left(\lambda_{1} \lambda_{2} \lambda_{3} \Lambda^{\beta}\right)\left[\frac{1}{2}\left(\xi_{1} \cdot \xi_{2}\right)\left(u+\frac{1}{4}\right)\left(s+\frac{1}{4}\right)\right] \\
& \times\left(\left(k_{3} \cdot k_{1}\right)^{n}\left(k_{3} \cdot k_{2}\right)^{m}+\left(k_{3} \cdot k_{1}\right)^{n}\left(k_{1} \cdot k\right)^{m}+\left(k \cdot k_{2}\right)^{m}\left(k \cdot k_{1}\right)^{n}+\left(k_{1} \cdot k\right)^{n}\left(k_{3} \cdot k_{1}\right)^{m}\right. \\
& \left.+\left(k_{3} \cdot k_{2}\right)^{m}\left(k_{2} \cdot k\right)^{n}+\left(k \cdot k_{2}\right)^{n}\left(k_{1} \cdot k\right)^{m}+\left(k_{3} \cdot k_{2}\right)^{n}\left(k_{1} \cdot k_{3}\right)^{m}+\left(k_{3} \cdot k_{2}\right)^{n}\left(k_{2} \cdot k\right)^{m}\right) .
\end{aligned}
$$

In (47) $k$ does indicate the off-shell tachyon's momentum. Note that in order to obtain this vertex we must consider both possible cases as the following :

$$
\operatorname{Tr}\left(\lambda_{3} \Lambda^{\beta} \lambda_{1} \lambda_{2}\right), \quad \operatorname{Tr}\left(\Lambda^{\beta} \lambda_{3} \lambda_{1} \lambda_{2}\right)
$$


Other cases have different coefficients, like $\operatorname{Tr}\left(\lambda_{2} \lambda_{1} \lambda_{3} \Lambda^{\beta}\right)$, and should not be computed as they made no contribution to the desired vertex. Because of the fact that we need some of the coefficients $a_{n, m}$ and $b_{n, m}$, let us address some of them as [6, 60]

$$
\begin{aligned}
& a_{0,0}=-\frac{\pi^{2}}{6}, b_{0,0}=-\frac{\pi^{2}}{12}, a_{1,0}=2 \zeta(3), a_{0,1}=0, b_{0,1}=-\zeta(3), a_{1,1}=a_{0,2}=-7 \pi^{4} / 90, \\
& a_{2,2}=\left(-83 \pi^{6}-7560 \zeta(3)^{2}\right) / 945, b_{2,2}=-\left(23 \pi^{6}-15120 \zeta(3)^{2}\right) / 1890, a_{1,3}=-62 \pi^{6} / 945, \\
& a_{2,0}=-4 \pi^{4} / 90, b_{1,1}=-\pi^{4} / 180, b_{0,2}=-\pi^{4} / 45, a_{0,4}=-31 \pi^{6} / 945, a_{4,0}=-16 \pi^{6} / 945, \\
& a_{1,2}=a_{2,1}=8 \zeta(5)+4 \pi^{2} \zeta(3) / 3, a_{0,3}=0, a_{3,0}=8 \zeta(5), b_{1,3}=-\left(12 \pi^{6}-7560 \zeta(3)^{2}\right) / 1890, \\
& a_{3,1}=\left(-52 \pi^{6}-7560 \zeta(3)^{2}\right) / 945, b_{0,3}=-4 \zeta(5), b_{1,2}=-8 \zeta(5)+2 \pi^{2} \zeta(3) / 3, \\
& b_{0,4}=-16 \pi^{6} / 1890 .
\end{aligned}
$$

where $b_{n, m}$ should be symmetric.

The following relations must be pointed out.

$$
k_{1} \cdot k=k_{2} \cdot k_{3}+\left(-k^{2}-m^{2}\right) / 2, \quad k_{2} \cdot k=k_{1} \cdot k_{3}+\left(-k^{2}-m^{2}\right) / 2
$$

Note that $-k^{2}-m^{2}$ in the vertex (47) will be removed with those common terms in the propagator and will give rise to some contact interactions of one RR, two scalar fields and one tachyon that we do not consider now. So for the moment we do over look them. In fact, we will observe that finally one has to subtract them from the interaction terms that concluded from the amplitude of one RR, two scalar fields and one tachyon. In the last section once again we try to come back to those terms. Having neglected them, one gets an infinite number of tachyon poles as :

$$
\begin{aligned}
& -32 i \pi \alpha^{\prime 2} \beta^{\prime} \mu_{p}^{\prime} \frac{\epsilon^{i_{0} \cdots i_{p}} H_{i_{0} \cdots i_{p}}}{(p+1) !\left(s^{\prime}+t+u^{\prime}\right)} \operatorname{Tr}\left(\lambda_{1} \lambda_{2} \lambda_{3}\right) \sum_{n, m=0}^{\infty}\left(\left(a_{n, m}+b_{n, m}\right)\left[s^{\prime m} u^{\prime n}+s^{\prime n} u^{\prime m}\right]\right. \\
& \left.\times\left[\left(\xi_{1} \cdot \xi_{2}\right) \frac{1}{2} u^{\prime} s^{\prime}\right]\right) .
\end{aligned}
$$

In order to check these proposed infinite couplings (43), we are going to compare (49) with the infinite number of the tachyon poles in string theory for several values of $n, m$. Note that we have removed the common factors in both string and field theory sides.

First we set, $n=m=0$, then (49) gives us a coefficient as :

$$
-8\left(a_{0,0}+b_{0,0}\right)=-8\left(\frac{-\pi^{2}}{6}+\frac{-\pi^{2}}{12}\right)=2 \pi^{2}
$$


On the other hand, we have a factor of $\left(2 \pi^{2} c_{0,0}\right)$ in (31). Comparing $\left(2 \pi^{2} c_{0,0}\right)$ with the factor of $\left(2 \pi^{2}\right)$ in field theory one gets the consistent result. Let us proceed at first order of $\alpha^{\prime}$, so (49) does include a factor as

$$
-4\left(a_{1,0}+a_{0,1}+b_{1,0}+b_{0,1}\right)\left(s^{\prime}+u^{\prime}\right)=0
$$

Equation (31) is also proportional to $\pi^{2}\left(c_{1,0}+c_{0,1}\right)\left(s^{\prime}+u^{\prime}\right)$, which is indeed zero. At second order or $\left(\alpha^{\prime}\right)^{2}$, (49) does involve a coefficient as

$$
\begin{aligned}
& -8\left(a_{1,1}+b_{1,1}\right) s^{\prime} u^{\prime}-4\left(a_{0,2}+a_{2,0}+b_{0,2}+b_{2,0}\right)\left[s^{\prime 2}+u^{\prime 2}\right] \\
& =\frac{\pi^{4}}{3}\left(2 s^{\prime} u^{\prime}\right)+\frac{2 \pi^{4}}{3}\left(s^{\prime 2}+u^{\prime 2}\right)
\end{aligned}
$$

Again (31) does have a factor $\pi^{2}\left[c_{1,1}\left(2 s^{\prime} u^{\prime}\right)+\left(c_{2,0}+c_{0,2}\right)\left(s^{\prime 2}+u^{\prime 2}\right)\right]$, which is precisely equivalent to string amplitude ( by making use of (25)). In order $\alpha^{\prime 3}$, (49) has the following coefficient

$$
\begin{aligned}
& -4\left(a_{3,0}+a_{0,3}+b_{0,3}+b_{3,0}\right)\left[s^{\prime 3}+u^{\prime 3}\right]-4\left(a_{1,2}+a_{2,1}+b_{1,2}+b_{2,1}\right)\left[s^{\prime} u^{\prime}\left(s^{\prime}+u^{\prime}\right)\right] \\
& =-16 \pi^{2} \xi(3) s^{\prime} u^{\prime}\left(s^{\prime}+u^{\prime}\right)
\end{aligned}
$$

and in (31) we have the following factor $\pi^{2}\left[\left(c_{0,3}+c_{3,0}\right)\left[s^{\prime 3}+u^{\prime 3}\right]+\left(c_{2,1}+c_{1,2}\right) s^{\prime} u^{\prime}\left(s^{\prime}+u^{\prime}\right)\right]$. Finally at order $\left(\alpha^{\prime}\right)^{4}$, (49) consists a factor of

$$
\begin{aligned}
& -4\left(a_{4,0}+a_{0,4}+b_{0,4}+b_{4,0}\right)\left(s^{\prime 4}+u^{\prime 4}\right)-4\left(a_{3,1}+a_{1,3}+b_{3,1}+b_{1,3}\right)\left[s^{\prime} u^{\prime}\left(s^{\prime 2}+u^{\prime 2}\right)\right] \\
& -8\left(a_{2,2}+b_{2,2}\right) s^{\prime 2} u^{\prime 2}=\frac{4 \pi^{6}}{15}\left(s^{\prime 4}+u^{\prime 4}+2\left(s^{\prime 3} u^{\prime}+u^{\prime 3} s^{\prime}\right)+3 s^{\prime 2} u^{\prime 2}\right)
\end{aligned}
$$

and (31) shows the factor of $\pi^{2}\left[\left(c_{4,0}+c_{0,4}\right)\left(s^{\prime 4}+u^{\prime 4}\right)+\left(c_{1,3}+c_{3,1}\right)\left(s^{\prime 3} u^{\prime}+u^{\prime 3} s^{\prime}\right)+2 c_{2,2} s^{\prime 2} u^{\prime 2}\right]$ which is surprisingly equal to the above factor making use of the coefficients in (25). One might conclude that these comparisons can be easily extended 4 to all orders of $\alpha^{\prime}$. Thus, (49) precisely does reproduce the infinite number of tachyon poles of the string amplitude of (31).

This shows that in addition to higher derivative couplings of two scalars and two tachyons are being exact up to on-shell ambiguity, the momentum expansion of the amplitude $C \phi \phi T$ does agree with $T T \phi \phi$ 's momentum expansion .

\footnotetext{
${ }^{4}$ Similar computations for the amplitude of one RR and three massless open strings have been checked in $[6,7,8]$.
} 


\section{$7 p=n+1$ case}

The trace in $\mathcal{A}_{2}$ amplitude can be done as follows

$$
\operatorname{Tr}\left(H_{(n)} M_{p} \Gamma^{b a}\right)= \pm \frac{32}{n !} \epsilon^{a_{0} \cdots a_{p-2} b a} H_{a_{0} \cdots a_{p-2}},
$$

Taken into account this trace in $\mathcal{A}_{2}$, one gets the amplitude in string side as

$$
\mathcal{A}^{\phi \phi T C}=\mp \frac{32 i}{(p-1) !}\left(\mu_{p}^{\prime} \beta^{\prime} \pi^{1 / 2}\right) \operatorname{Tr}\left(\lambda_{1} \lambda_{2} \lambda_{3}\right) H_{a_{0} \cdots a_{p-2}} \epsilon^{a_{0} \cdots a_{p-2} b a}\left\{2 k_{1 a} k_{2 b} \xi_{1} \cdot \xi_{2} L_{2}\right\} .
$$

As it is obvious from (50) the amplitude is antisymmetric with respect to two scalar fields. It shows that the amplitude must have zero value just for the Abelian gauge. The amplitude also does have an infinite number of massless poles in the t-channel and an infinite number of contact interactions. First we produce all infinite numbers of massless poles and then we come to all contact interactions. Now we want to make an important comment. Because of some kinematic reason in our amplitude we do not have any tachyon pole and this is unlike the scattering amplitude of two gauge fields, one RR and one tachyon so we do not have any tachyon pole in field theory either.

\subsection{Infinite number of massless poles and contact interactions for $p=n+1$ case}

Having replaced the expansion of $L_{2}$ and the related trace into $\mathcal{A}_{2}$, one comes to an infinite number of the massless poles in the $t$-channel as

$$
\begin{aligned}
\mathcal{A}^{\phi \phi T C}= & \pm \frac{32 i \mu_{p}^{\prime} \beta^{\prime} \pi^{2}}{t(p-1) !} \operatorname{Tr}\left(\lambda_{1} \lambda_{2} \lambda_{3}\right) H_{a_{0} \cdots a_{p-2}} \epsilon^{a_{0} \cdots a_{p} b a} \sum_{n=-1}^{\infty} b_{n}(u+s+1 / 2)^{n+1} \\
& \times\left[2 \xi_{1} \cdot \xi_{2} k_{1 a} k_{2 b}\right]
\end{aligned}
$$

There are also an infinite number of contact interactions for this case that we want to consider later on. In field theory, these massless poles should be read off with this Feynman diagram

$$
\mathcal{A}=V_{\alpha}^{i}\left(C_{p-2}, T_{3}, A\right) G_{\alpha \beta}^{i j}(A) V_{\beta}^{j}\left(A, \phi_{1}, \phi_{2}\right),
$$

As observed from the expansion of the amplitude, there are an infinite number of higher derivative couplings between one $\mathrm{RR}(\mathrm{p}-2)$-form field $\left(C_{p-2}\right)$, one on-shell tachyon and one off-shell gauge field where they are related to the higher derivative corrections of the WZ 
coupling $\operatorname{Tr}\left(C_{p-2} \wedge F \wedge D T\right)$. They have been found in [23]. Therefore the vertex of $V_{\alpha}^{i}\left(C_{p-2}, T_{3}, A\right)$ must be found from the higher derivative corrections of this WZ coupling such that gauge field has to be off-shell. Thus the needed vertices and gauge field propagator are given as

$$
\begin{aligned}
V_{\alpha}^{i}\left(C_{p-2}, T_{3}, A\right) & =2 \mu_{p}^{\prime} \beta^{\prime}\left(2 \pi \alpha^{\prime}\right)^{2} \frac{1}{(p-1) !} \epsilon^{a_{0} \cdots a_{p-1} i} H_{a_{0} \cdots a_{p-2}} k_{a_{p-1}} \sum_{n=-1}^{\infty} b_{n}\left(\alpha^{\prime} k_{3} \cdot k\right)^{n+1} \operatorname{Tr}\left(\lambda_{3} \Lambda^{\alpha}\right), \\
V_{\beta}^{j}\left(A, \phi_{1}, \phi_{2}\right) & =-i T_{p}\left(2 \pi \alpha^{\prime}\right)^{2} \xi_{1} \cdot \xi_{2}\left(k_{1}-k_{2}\right)^{j}\left[-\operatorname{Tr}\left(\lambda_{1} \lambda_{2} \Lambda_{\beta}\right)+\operatorname{Tr}\left(\lambda_{2} \lambda_{1} \Lambda_{\beta}\right)\right], \\
G_{\alpha \beta}^{i j}(A) & =\frac{i \delta_{\alpha \beta} \delta^{i j}}{\left(2 \pi \alpha^{\prime}\right)^{2} T_{p}(t)}
\end{aligned}
$$

where the gauge field propagator must be found from its kinetic term, which is given in (26). On the other hand, the vertex of $V_{\beta}^{j}\left(A, \phi_{1}, \phi_{2}\right)$ has been reduced from the scalar field's kinetic term $\frac{\lambda^{2}}{2} \operatorname{Tr}\left(D_{a} \phi_{i} D^{a} \phi^{i}\right)$. The other remark that should be made is that, in order to produce the desired amplitude for 123 ordering we must keep just the first term in the vertex of $V_{\beta}^{j}\left(A, \phi_{1}, \phi_{2}\right)$. As always $k$ is the off-shell gauge field's momentum. Regarding the point that massless poles of amplitude have no higher derivative corrections, one may understand that scalar field's kinetic term does not involve correction so the vertex $V_{\beta}^{j}\left(A, \phi_{1}, \phi_{2}\right)$ does not have a higher derivative correction either, because it has already been fixed in the DBI action.

One replaces (53) into (52), to get

$$
\begin{aligned}
\mathcal{A}= & \left(2 \pi \alpha^{\prime}\right)^{2} \frac{2 \mu_{p}^{\prime} \beta^{\prime}}{(p-1) ! t} \epsilon^{a_{0} \cdots a_{p-1} j} H_{a_{0} \cdots a_{p-2}} \operatorname{Tr}\left(\lambda_{1} \lambda_{2} \lambda_{3}\right) \sum_{n=-1}^{\infty} b_{n}\left(\frac{\alpha^{\prime}}{2}\right)^{n+1}(s+u+1 / 2)^{n+1} \\
& \times\left(-2\left(\xi_{1} \cdot \xi_{2}\right) k_{2 a_{p-1}} k_{1 j}\right) .
\end{aligned}
$$

which is precisely the infinite number of the t-channel massless poles of (51). As a comment and unlike $p=n-1$ case, here there should not be any residual contact interactions in (54). Indeed we get this result after comparing all massless poles of field theory (54) with the infinite number of massless poles in string side.

This does show that the momentum expansion of our amplitude $C \phi \phi T$ is actually consistent, even with the momentum expansion of $C A T$. Let us end this section by producing all infinite numbers of contact terms for $p=n+1$ case in field theory .

Having replaced $L_{2}$ into the S-matrix in $\mathcal{A}_{2}$, one gets contact terms at all orders of $\alpha^{\prime}$ in string theory as

$$
\mathcal{A}^{\phi \phi T C}=\mp \frac{32 i}{(p-1) !}\left(\mu_{p}^{\prime} \beta^{\prime} \pi^{2}\right) \operatorname{Tr}\left(\lambda_{1} \lambda_{2} \lambda_{3}\right) H_{a_{0} \cdots a_{p-2}} \epsilon^{a_{0} \cdots a_{p-2} b a}
$$




$$
\times\left(2 k_{1 a} k_{2 b} \xi_{1} \cdot \xi_{2}\right)\left(\sum_{p, n, m=0}^{\infty} e_{p, n, m} t^{p}\left(s^{\prime} u^{\prime}\right)^{n}\left(s^{\prime}+u^{\prime}\right)^{m}\right) .
$$

These infinite numbers of contact terms in (55) can be reproduced by taking the following gauge invariant couplings:

$$
\begin{aligned}
S_{1}= & 2 i \lambda^{2} \beta^{\prime} \mu_{p}^{\prime} \int d^{p+1} \sigma \frac{1}{(p-2) !}\left(\varepsilon^{v}\right)^{a_{0} \cdots a_{p}} \sum_{p, n, m=0}^{\infty} e_{p, n, m}\left(\alpha^{\prime}\right)^{2 n+m+1}\left(\frac{\alpha^{\prime}}{2}\right)^{p} \\
& \times C_{a_{0} \cdots a_{p-3}}^{(p-2)}(\sigma) \operatorname{Tr}\left(D^{a_{1}} \cdots D^{a_{2 n}} D^{b_{1}} \cdots D^{b_{m}} D_{a_{p-2}} T\right. \\
& \left.\left(D^{a} D_{a}\right)^{p} D_{b_{1}} \cdots D_{b_{m}}\left[D_{a_{1}} \cdots D_{a_{n}} D_{a_{p-1}} \phi^{i} D_{a_{n+1}} \cdots D_{a_{2 n}} D_{a_{p}} \phi_{i}\right]\right) .
\end{aligned}
$$

Here $\left(\varepsilon^{v}\right)$ is the volume form which takes place in subspace parallel to the brane's world volume. Note that, to produce (55), one may be able to write down another higher derivative gauge invariant couplings, to make a contribution to the contact terms of $C \phi \phi T$ amplitude. Thus (56) is in fact one special higher derivative gauge invariant coupling that results all terms in (55).

So we get to an important remark. The leading order terms of our amplitude related to Wess-Zumino couplings and naturally higher order terms must correspond to WZ couplings' higher derivatives .

Therefore we learned that by analyzing massless poles we get useful information about the higher derivative corrections of $\operatorname{Tr}\left(C_{p-2} \wedge F \wedge D T\right)$. By studying contact interactions we gain remarkable information on new coupling as $\operatorname{Tr}\left(C_{p-2} \wedge D T \wedge D \phi \wedge D \phi\right)$.

It is worth talking about some details related to WZ couplings. They can be derived also with BSFT method. However as noted in [17] setting constant RR field reduces to having no higher derivative correction to these WZ couplings.

Notice the point that we have already mentioned. Our derived couplings make sense in the presence of $p_{a} p^{a} \rightarrow \frac{1}{4}$. So the conclusion as a matter of fact is that we are not allowed to compare our couplings with the constant $\mathrm{RR}$ field as a $\left(p_{a} p^{a}=0\right)$ result of the BSFT.

\subsection{Contact terms}

Doing in detail all an infinite number of tachyon and massless poles of string theory amplitude (19), we are now ready to extract the rest of the contact terms of the amplitude. As can be seen from the poles of the Gamma function, $\left(-t-s^{\prime}-u^{\prime}\right) L_{1}$ has neither a massless 
pole nor a tachyon pole. We will show now this consistency with the WZ terms . By setting $n=p+1$ to all terms in $A_{1}$ in equation (19) except the last term, the string scattering amplitude takes the following form:

$$
\begin{aligned}
A_{1}^{C \phi \phi T}= & \frac{32 i \pi^{3} \mu_{p}^{\prime} \beta^{\prime}}{(p+1) !} \xi_{1}^{i} \xi_{2}^{j}\left(-p_{i} p_{j}\left(H^{(p+1)}\right)_{a_{0} \cdots a_{p}}\right. \\
& +(p+1) k_{1 a_{0}} p_{j}\left(H^{(p+1)}\right)_{i a_{1} \cdots a_{p}}+(p+1) k_{2 a_{0}} p_{i}\left(H^{(p+1)}\right)_{j a_{1} \cdots a_{p}} \\
& \left.+p(p+1) k_{1 a_{0}} k_{2 a_{1}}\left(H^{(p+1)}\right)_{i j a_{2} \cdots a_{p}}\right)\left(\varepsilon^{v}\right)^{a_{0} \cdots a_{p}} \\
& \times\left(\sum_{n=0}^{\infty} c_{n}\left(s^{\prime}+t+u^{\prime}\right)^{n+1}+\sum_{n, m=0}^{\infty} c_{n, m}\left[\left(s^{\prime}\right)^{n}\left(u^{\prime}\right)^{m}+\left(s^{\prime}\right)^{m}\left(u^{\prime}\right)^{n}\right]\right. \\
& \left.+\sum_{p, n, m=0}^{\infty} f_{p, n, m}\left(s^{\prime}+t+u^{\prime}\right)^{p+1}\left[\left(s^{\prime}+u^{\prime}\right)^{n}\left(s^{\prime} u^{\prime}\right)^{m}\right]\right)
\end{aligned}
$$

where $H^{(p+1)}=d C^{(p)}$. In fact, by analyzing the $C \phi \phi T$ amplitude, one understands that it needs some interaction terms in which either must come from pullback or Taylor expansion of one RR p-form field $\left(C^{(p)}\right)$. Due to not having any external gauge field here we just replaced all covariant derivative of scalars and tachyon with their partial derivatives.

One may check that the leading contact terms in our amplitude can be reproduced by considering the following field interactions:

$$
\begin{aligned}
S_{2}=\frac{\lambda^{3} \beta^{\prime} \mu_{p}^{\prime}}{2 p !} \int d^{p+1} & \sigma\left(\varepsilon^{v}\right)^{a_{0} \cdots a_{p}}\left(\operatorname{Tr}\left(\partial_{a_{0}} T \Phi^{i} \Phi^{j}\right) \partial_{i} \partial_{j} C_{a_{1} \cdots a_{p}}^{(p)}\right. \\
& +2 p \operatorname{Tr}\left(\partial_{a_{0}} T \partial_{a_{1}} \Phi^{i} \Phi^{j}\right) \partial_{j} C_{i a_{2} \cdots a_{p}}^{(p)} \\
& \left.+(p-1) p \operatorname{Tr}\left(\partial_{a_{0}} T \partial_{a_{1}} \Phi^{i} \partial_{a_{2}} \Phi^{j}\right) C_{i j a_{3} \cdots a_{p}}^{(p)}\right)
\end{aligned}
$$

Applying integration by parts, these contributions (58) might be reconsidered as

$$
\begin{gathered}
S_{2}=\frac{\lambda^{3} \beta^{\prime} \mu_{p}^{\prime}}{2 p !} \int d^{p+1} \sigma\left(\varepsilon^{v}\right)^{a_{0} \cdots a_{p}}\left(\operatorname{Tr}\left(\partial_{a_{0}} T \Phi^{i} \Phi^{j}\right) \partial_{j} H_{i a_{1} \cdots a_{p}}^{(p+1)}\right. \\
\left.+p \operatorname{Tr}\left(\partial_{a_{0}} T \partial_{a_{1}} \Phi^{i} \Phi^{j}\right) H_{i j a_{2} \cdots a_{p}}^{(p+1)}\right)
\end{gathered}
$$

where for higher derivative corrections our notation is as follows:

$$
\begin{aligned}
\left(s^{\prime} u^{\prime}\right)^{m} H T \phi \phi & =\left(\alpha^{\prime}\right)^{2 m} H D_{a_{1}} \cdots D_{a_{2 m}} T \partial^{a_{1}} \cdots \partial^{a_{m}} \phi \partial^{a_{m+1}} \cdots \partial^{a_{2 m}} \phi, \\
\left(s^{\prime}+u^{\prime}\right)^{n} H T \phi \phi & =\left(\alpha^{\prime}\right)^{n} H D_{a_{1}} \cdots D_{a_{n}} T \partial^{a_{1}} \cdots \partial^{a_{n}}(\phi \phi), \\
\left(s^{\prime}\right)^{n} u^{\prime m} H T \phi \phi & =\left(\alpha^{\prime}\right)^{n+m} H D_{a_{1}} \cdots D_{a_{n}} D_{a_{1}} \cdots D_{a_{m}} T \partial^{a_{1}} \cdots \partial^{a_{n}} \phi \partial^{a_{1}} \cdots \partial^{a_{m}} \phi, \\
\left(s^{\prime}+t+u^{\prime}\right)^{p+1} H T \phi \phi & =\left(\frac{\alpha^{\prime}}{2}\right)^{p+1} H\left(D_{a} D^{a}\right)^{p+1}(T \phi \phi) .
\end{aligned}
$$


Now we write down the contact terms that are related to the last term of $A_{1}$ in (19). Note that it is not possible to have a massless scalar field as an off-shell state. Because if we imagine there was a nonvanishing coupling between one scalar field and one RR ( $p+1)$-form field strength then the interaction in WZ action would be

$$
\lambda \mu_{p}^{\prime} \int d^{p+1} \sigma \frac{1}{(p+1) !}\left(\varepsilon^{v}\right)^{a_{0} \cdots a_{p}} \operatorname{Tr}\left(\Phi^{i}\right) H_{i a_{0} \cdots a_{p}}^{(p+2)}(\sigma)
$$

Therefore this off-shell scalar created by that interaction should be attached within an interaction including two on-shell scalars and one on-shell tachyon. However, there are no world-volume interactions between three scalars and one tachyon. The same thing happens for the case of an off-shell gauge field because it will not have any coupling with the RR $(\mathrm{p}+1)$-form field. Thus the amplitude for $n=p+1$ does not involve any scalar/gauge pole.

Extracting the trace and keeping just the contact terms for the last term in $A_{1}$ we end up with the following terms

$$
\begin{aligned}
A_{1}^{c \phi \phi T}= & \frac{16 i \pi^{3} \mu_{p}^{\prime} \beta^{\prime}}{(p+1) !} \xi_{1} \cdot \xi_{2}\left(\left(H^{(p+1)}\right)_{a_{0} \cdots a_{p}}\left(\varepsilon^{v}\right)^{a_{0} \cdots a_{p}}\right)\left(\sum_{n=0}^{\infty} c_{n}\left(s^{\prime}+t+u^{\prime}\right)^{n} s^{\prime} u^{\prime}\right. \\
& \left.+\sum_{p, n, m=0}^{\infty} f_{p, n, m}\left(s^{\prime}+t+u^{\prime}\right)^{p}\left[\left(s^{\prime}+u^{\prime}\right)^{n}\left(s^{\prime} u^{\prime}\right)^{m+1}\right]\right),
\end{aligned}
$$

The above contact terms in this part of the amplitude are now a sort of new couplings which can be produced by the following field interactions in field theory as well:

$$
S_{3}=\frac{i \lambda^{3} \beta^{\prime} \mu_{p}^{\prime}}{4 p !} \int d^{p+1} \sigma\left(\varepsilon^{v}\right)^{a_{0} \cdots a_{p}}\left(\operatorname{Tr}\left(\partial_{a_{0}} T \Phi^{i} \Phi_{i}\right) C_{a_{1} \cdots a_{p}}^{(p)}\right),
$$

Contact terms in the first line of (61) will be reproduced by taking the following couplings:

$$
-\lambda^{3} \beta^{\prime} \mu_{p}^{\prime} \sum_{n=0}^{\infty} c_{n}\left(\frac{\alpha^{\prime}}{2}\right)^{n} C_{p} \wedge\left(D^{a} D_{a}\right)^{n}\left[D^{a} D^{b} D T\left(D_{a} \phi^{i} D_{b} \phi_{i}\right)\right],
$$

It becomes so clear from (63) that the nonleading order terms do correspond to the higher derivative corrections of (62). Indeed it looks like the coupling which we found in non-BPS formalism $C_{p} \wedge D T T^{2}$. The contact terms in the second line of (61) might correspond to a coupling such as

$$
\begin{aligned}
& -\lambda^{3} \beta^{\prime} \mu_{p}^{\prime} \sum_{p, n, m=0}^{\infty} f_{p, n, m}\left(\alpha^{\prime}\right)^{2 m+n}\left(\frac{\alpha^{\prime}}{2}\right)^{p} H_{p+1}\left(D_{a} D^{a}\right)^{p} \\
& {\left[D_{a} D_{b} D^{a_{1}} \cdots D^{a_{n}} D_{b_{1}} \cdots D_{b_{2 m}} T D_{a_{1}} \cdots D_{a_{n}}\left(D^{a} D^{b_{1}} \cdots D^{b_{m}} \phi^{i} D^{b} D^{b_{m+1}} \cdots D^{b_{2 m}} \phi_{i}\right)\right]}
\end{aligned}
$$


These new interactions in field theory are completely consistent with the string theory amplitude of one RR $p$ - form field, two scalar fields and one tachyon. Therefore, it shows up that perturbative string theory is a strong tool to discover new couplings in field theory side.

Eventually let us come back to the contact interaction terms that amplitude (45) have resulted. Applying some identities, we write down all contact interactions as:

$$
\begin{aligned}
& 32 i \pi \beta^{\prime} \mu_{p}^{\prime} \frac{\epsilon^{a_{0} \cdots a_{p}} H_{a_{0} \cdots a_{p}}}{(p+1) !}\left[\left(\xi_{1} \cdot \xi_{2}\right) \frac{1}{2} u^{\prime} s^{\prime}\right] \sum_{n, m=0}^{\infty}\left(a_{n, m}+b_{n, m}\right)\left(-\alpha^{\prime} k^{2}-\alpha^{\prime} m^{2}\right)^{l-1} \\
& {\left[\left(4 \sum_{l=1}^{m}\left(\begin{array}{c}
m \\
l
\end{array}\right)\left(s^{\prime m-l} u^{\prime n}+u^{\prime m-l} s^{\prime n}\right)+4 \sum_{l=1}^{n}\left(\begin{array}{c}
n \\
l
\end{array}\right)\left(s^{\prime n-l} u^{\prime m}+u^{\prime n-l} s^{\prime m}\right)\right)\right.} \\
& \left.+\sum_{l=1, j=1}^{n, m}\left(\begin{array}{c}
n \\
l
\end{array}\right)\left(\begin{array}{c}
m \\
j
\end{array}\right)\left(s^{\prime n-l} u^{\prime m-j}+u^{\prime n-l} s^{\prime m-j}\right)\left(-\alpha^{\prime} k^{2}-\alpha^{\prime} m^{2}\right)^{j}\right] \operatorname{Tr}\left(\lambda_{1} \lambda_{2} \lambda_{3}\right)
\end{aligned}
$$

Notice that these interactions could be reconsidered in the following form:

$32 i \pi \beta^{\prime} \mu_{p}^{\prime} \frac{\epsilon^{a_{0} \cdots a_{p}} H_{a_{0} \cdots a_{p}}}{(p+1) !}\left[\left(\xi_{1} \cdot \xi_{2}\right) \frac{1}{2} u^{\prime} s^{\prime}\right] \operatorname{Tr}\left(\lambda_{1} \lambda_{2} \lambda_{3}\right) \sum_{p, n, m=0}^{\infty} f_{p, n, m}^{\prime}\left(s^{\prime}+t+u^{\prime}\right)^{p}\left(s^{\prime}+u^{\prime}\right)^{n}\left(s^{\prime} u^{\prime}\right)^{m},(65$

One may be able to write $f_{p, n, m}^{\prime}$ in terms of $a_{n, m}$ and $b_{n, m}$. One has to consider the fact that the last contact terms in (23) have the same structure as those terms appeared in (65). Thus the coefficients $f_{p, n, m}$ in (64) must be substituted with

$$
f_{p, n, m} \rightarrow f_{p, n, m}-f_{p, n, m}^{\prime}
$$

Therefore the higher derivative theory will exactly give rise to the string theory amplitude.

\subsection{Contact terms for $n=p+3$}

For this case which includes a $R R(p+2)$-form field, one gets the fact that exchanging a massless gauge/scalar is not allowed. In particular, the $(p+2)$-form potential has one rank more than $R R(p+1)$-form potential to result in a desired interaction in world volume space. Thus for this case we might expect that our amplitude does not involve any massless/tachyon poles. Thus the leading terms of the amplitude are in fact just contact terms that we want to identify now. The infinite number of contact terms in string amplitude is given as

$$
\mathcal{A}_{3}=\frac{32 i \pi^{2} \mu_{p}^{\prime} \beta^{\prime}}{(p+3) !}\left(H^{(p+3)}\right)_{i j a_{0} \cdots a_{p}} \xi_{1 i} \xi_{2 j}\left(\varepsilon^{v}\right)^{a_{0} \cdots a_{p}}
$$




$$
\times\left(\sum_{n=-1}^{\infty} b_{n}\left(u^{\prime}+s^{\prime}\right)^{n+1}+\sum_{p, n, m=0}^{\infty} e_{p, n, m} t^{p+1}\left(s^{\prime} u^{\prime}\right)^{n}\left(s^{\prime}+u^{\prime}\right)^{m}\right) .
$$

In order to reproduce all terms in (66) we begin with the WZ action. The minimal interaction for this case does include a $R R(p+2)$-form field in the bulk, two scalars within commutator and one on-shell tachyon. Thus the interaction that does include the RR $(p+2)$-form potential is

$$
S_{4}=\frac{\left(2 \pi \alpha^{\prime}\right) \lambda \beta^{\prime} \mu_{p}^{\prime}}{p !} \int d^{p+1} \sigma\left(\varepsilon^{v}\right)^{a_{0} \cdots a_{p}}\left(\operatorname{Tr}\left(\partial_{a_{0}} T\left[\Phi^{i}, \Phi^{j}\right]\right)\right) C_{j i a_{1} \cdots a_{p}}^{(p+2)},
$$

so by extracting commutator we get the following leading nonvanishing coupling which is confirmed by direct S-matrix computations as follows:

$$
S_{4}=\frac{\left(2 \pi \alpha^{\prime}\right) 2 \lambda \beta^{\prime} \mu_{p}^{\prime}}{p !} \int d^{p+1} \sigma\left(\varepsilon^{v}\right)^{a_{0} \cdots a_{p}}\left(\operatorname{Tr}\left(\partial_{a_{0}} T \Phi^{i} \Phi^{j}\right)\right) C_{j i a_{1} \cdots a_{p}}^{(p+2)},
$$

where for higher derivative corrections our notation is as follows:

$$
(t)^{p} H T \phi \phi=\left(\frac{\alpha^{\prime}}{2}\right)^{p} H T\left(D_{a} D^{a}\right)^{p}(\phi \phi) .
$$

So we have produced the higher derivative theory with the string theory amplitude of $C \phi \phi T$. It is now fair to say that we have shown the complete consistency of one tachyon, two scalars and one closed string RR around (22) with all its higher derivative couplings in field theory.

This ends our goal which was to show complete consistency between string theory scattering amplitudes for different values of $p, n$ and making use of symmetrized trace tachyon DBI action.

\section{Concluding remarks}

As it is seen for the simple tachyon poles, the coupling of $\operatorname{Tr}\left(C_{p} \wedge D T\right)$ does not get any correction. Therefore by studying nonleading tachyon poles one can find information about the higher derivative corrections to the coupling of two scalar fields and two tachyons where we found them up to all orders of $\alpha^{\prime}$ in (43). This is worth mentioning that contact terms of the string amplitude already include information about the higher derivative corrections to $\operatorname{Tr}\left(C_{p-2} \wedge D T \wedge D \phi^{i} \wedge D \phi_{i}\right), \operatorname{Tr}\left(C_{p} \wedge D T \phi^{i} \phi_{i}\right)$ and $\operatorname{Tr}\left(\partial_{a_{0}} T\left[\Phi^{i}, \Phi^{j}\right]\right) C_{j i a_{1} \cdots a_{p}}^{(p+2)}\left(\varepsilon^{v}\right)^{a_{0} \cdots a_{p}}$. Note that here we obtained a new coupling between the commutator of scalar fields with 
covariant derivative of tachyon and one RR. It is an interesting issue to study the scattering amplitude between one RR, one tachyon, one gauge field and one scalar field in the world volume of non-BPS branes to get more information on WZ couplings. It would be also nice to follow this amplitude in order to get all the information needed about the higher derivative corrections of this amplitude to WZ and tachyonic actions [61].

\subsection{Discussion}

We have done the S-matrix elements of $C T \phi \phi$ in the world volume of a single non-BPS SD-brane. Having set the on-shell conditions, we believe that $C T \phi \phi$ amplitude makes sense just in the presence of a single non-BPS SD-brane. Applying its momentum expansion, we were able to show the consistency of the leading order terms of the expansion with the WZ couplings of a single non-BPS SD-brane. The non-leading terms have been extracted with the help of some higher derivative corrections of the WZ couplings. In fact, they have been

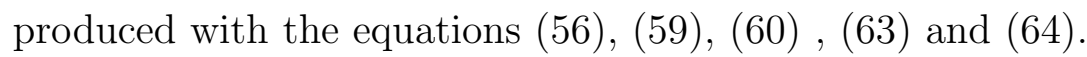

The amplitude of $C \phi \phi T$ consists of two parts. The first part does include a $\mathrm{RR} p$-form $\left(C_{p}\right)$ with an infinite number of tachyon poles and many contact interaction terms. The contact interactions give rise to a new coupling of the form $\operatorname{Tr}\left(C_{p} \wedge D T \phi^{i} \phi_{i}\right)$ and give some higher derivative corrections to this coupling. They are in precise agreement with S-matrix elements of this amplitude for $p+1=n$ case. To reproduce an infinite number of tachyon poles, one has to find two tachyon and two scalar field couplings to all orders of $\alpha^{\prime}$ where we were able to find them in (43) with direct S-matrix computations. We could confirm and check them with tachyon poles in our S-matrix computations.

Note also that comparing an infinite number of field theory tachyon poles with the infinite poles of string theory gave rise to some residual contact interactions. Due to having the same structure for these contact interactions as those that appeared in the second line of (23), we should be able to modify the coefficients $f_{p, n, m}$ in (64). Therefore one must substitute $f_{p, n, m}-f_{p, n, m}^{\prime}$ instead of $f_{p, n, m}$ in (64).

The second part does contain a RR (p-2)-form field $\left(C_{p-2}\right)$ with an infinite number of massless poles and many contact terms. All massless poles must be reproduced by the higher derivative couplings of one $\mathrm{RR}(p-2)$-form field, one Abelian field strength and covariant derivative of the tachyon or in the other words with the coupling of $\operatorname{Tr}\left(C_{p-2} \wedge\right.$ 
$D T \wedge F)$ and by making use of kinetic term of scalar fields. One may point out that in this part of the amplitude we have to use the commutator of the scalar and gauge field as well. In order to reproduce contact terms of $C \phi \phi T$ for $p=n+1$ case, the coupling of $\operatorname{Tr}\left(C_{p-2} \wedge D T \wedge D \phi_{i} \wedge D \phi^{i}\right)$ and its higher derivative corrections must be taken into account. We have also fixed the coefficient of this coupling.

In the present paper, making use of direct string computations, we have confirmed the existence of the commutator of scalars $\left[\Phi^{i}, \Phi^{j}\right]$ which could come from either the exponential inside of the WZ action, or the expansion of the $\operatorname{det}(Q)$ in the non-Abelian DBI action, where for this amplitude they come from the exponential within the WZ action.

Indeed to produce all infinite numbers of massless poles of the amplitude for $p=n+1$ case we had to use commutator of guage and scalar in order to have the vertex of two on-shell scalars and one off-shell gauge field where the contribution is coming from the commutator in the definition of $D_{a} \Phi^{i}$.

It is really important to highlight the point that we could find out a very interesting coupling between the commutator of two scalar fields and the partial derivative of the tachyon and one RR as the following:

$$
S_{4}=\frac{\left(2 \pi \alpha^{\prime}\right) \lambda \beta^{\prime} \mu_{p}^{\prime}}{p !} \int d^{p+1} \sigma\left(\varepsilon^{v}\right)^{a_{0} \cdots a_{p}}\left(\operatorname{Tr}\left(\partial_{a_{0}} T\left[\Phi^{i}, \Phi^{j}\right]\right)\right) C_{j i a_{1} \cdots a_{p}}^{(p+2)},
$$

Of course in this amplitude we just had one external tachyon and two scalars and one $\mathrm{RR}$, that is why we drop the commutator between tachyon and gauge field in the above coupling in the definition of DT and just considered the partial derivative of tachyon. We may suppose that in the above coupling perhaps there should be a covariant derivative of the tachyon instead of its partial derivative. It would be nice to confirm this coupling by doing a six-point function $C T A \phi \phi$, which we leave it for future work [27].

One more remarkable thing is that these potential interactions do depend strongly on the field strength of the RR. Thus the gauge invariance for the RR must be held as well. However, as it is clear from the Wess-Zumino action (3), all interaction terms were written in terms of $\mathrm{RR}$ potentials or just $C$ terms so one might realize that the $\mathrm{RR}$ gauge invariance has not been satisfied any more. However, for the interactions appeared in this paper, we were able to show that both representations for $\mathrm{RR}$ couplings do agree and the difference between them is some total derivative terms.

Another important comment should be made is as follows. Having taken integration by 
parts we arrive at some complicated couplings between terms which indeed have different notions. For example the terms coming from the interior product inside WZ action or the terms coming from the Taylor expansion or the the terms coming from pullback are completely mixed in applying the expansion of the Wess-Zumino action.

It is shown that the expansion of the amplitude of one RR, one tachyon and two scalar fields $C \phi \phi T$ around (22) does belong to the higher derivative correction of the Wess-Zumino terms. Therefore we truly believe that (22) is a unique momentum expansion of four-point functions including one $\mathrm{RR}$ in the bulk $(C)$, one tachyon $(T)$ and either two scalar fields $(\phi \phi)$ or two gauge fields $(A A)$. Notice that the leading order term in the amplitude does correspond to the mentioned effective actions and all nonleading terms of the amplitude are consistent with the higher derivative corrections of the effective actions. On general grounds we realize that this expansion also holds for $C T A \phi$ amplitude [27].

Let us compare (22) with the momentum expansion of the S-matrix elements including three massless open strings [6, 7, 8] and one RR closed string. Momentum expansion for these cases has been found [6], which is $\alpha^{\prime} k_{i} \cdot k_{j} \rightarrow 0$. In fact it is equal to $\alpha^{\prime}\left(k_{i}+k_{j}\right)^{2} \rightarrow 0$. While, if we include tachyon to the amplitude then we can conclude that the expansion around $\alpha^{\prime} k_{i} \cdot k_{j} \rightarrow 0$ for the tachyon for sure is not the same as $\alpha^{\prime}\left(k_{i}+k_{j}\right)^{2} \rightarrow 0$.

One important facet of these couplings is that they do include on-shell ambiguity. To resolve it, one has to compare it with some off-shell interactions which already appeared in BSFT formalism. It was seen in [17] that the WZ couplings are being exact once one considers the RR field as a constant field. Therefore, we have to write all interactions in the momentum space in terms of the Mandelstam variables such that now they will be written in terms of Ramond-Ramond's momentum. The Mandelstam variables for the amplitude of $C T \phi \phi$ must be sent to $t \rightarrow 0, s \rightarrow-1 / 4$ and $u \rightarrow-1 / 4$. Now, one may be able to reconsider them as $\left(p^{2}+2 k_{1} \cdot p\right) \rightarrow 0,\left(p^{2}+2 k_{2} \cdot p\right) \rightarrow 0$ and $\left(p^{2}+2 k_{3} \cdot p\right) \rightarrow \frac{-1}{4}$. Applying a constant RR field for these forms of the couplings one immediately concludes that there are no contributions for all higher derivative corrections.

However, once more remember that for non-BPS SD-branes, those couplings make sense just in the appearance of an extra assumption which is $p_{a} p^{a} \rightarrow 1 / 4$. Thus we are not allowed to compare our higher derivative couplings with the interactions coming from BSFT.

Note that the couplings $T T \phi \phi$ in (43) might have on-shell ambiguity which means that 
by replacing $m^{2} T$ with $D D T$ tachyon poles will not change but rather would produce some more additional contact interactions. Thus by performing the amplitude of two tachyons, two scalar fields and one gauge field in which couplings (43) would appear in tachyon poles and in contact interactions of this amplitude, we might solve all those ambiguities. It would be interesting to carry out this rather long but straightforward computation [61].

Let us address some more unsolved problems, where one of them has been already addressed in [22].

Therefore an interesting amplitude to solve in favor of symmetrized trace is the scattering amplitude of CTTTT. In order to investigate whether symmetric trace works for tachyon DBI action or ordinary trace, one must study in detail the S-matrix element of one closed string Ramond-Ramond and four tachyons in the system of brane-antibrane in which the infinite number of the simple tachyon poles have to result in the following Feynman diagram

$$
A=V^{\alpha}\left(C_{P-1}, T, T\right) G_{\alpha \beta}(T) V^{\beta}(T, T, T, T)
$$

This amplitude will solve this apparent ambiguity for the tachyon action. In favor of applying symmetric trace it is really a good test to follow this computation within detail.

As we have already observed extracting the higher order contact interactions is really extremely tedious. We have made some progress in finding full consistency of the scattering amplitudes including one closed string RR, one tachyon and two scalar fields in the world volume of a single non-BPS SD-brane. It is evident from other investigations that commutator terms play the key role at higher orders. It would be nice to check the details of some six-point functions in order to remove some ambiguities [62].

Symmetrized trace was informed in [63] to describe low energy gauge theory with some simple background fields. It was shown that this kind of trace prescription does need corrections at sixth order in gauge field's field strength [64]. In non-Abelian gauge theory these problems have been addressed in terms of the ambiguity between interchanging field strengths and covariant derivatives. One imagines that the commutators of field strengths might be redefined in terms of their covariant derivatives. Thus it would be interesting to discover within detail some six point functions to find interactions including some high derivatives of the gauge field's field strengths 62. Some progresses have been made [65], also through applying ideas of non commutative field theory some investigations had been 
done [66].

Let us address the final point. In order to get more information in finding higher derivative corrections for both tachyonic DBI and Wess-Zumino effective actions it is really a very nice issue to pursue the amplitude of one closed string RR field, one gauge field, one scalar field and one tachyon in the world volume of non-BPS branes [61].

\section{Acknowledgments}

The author wishes to thank K.S.Narain, F.Quevedo, N.Arkani-Hamed and I.Y. Park for helpful conversations. He would also like to thank J.Maldacena for comments and E.Martinec for useful suggestions. He also acknowledges L. Álvarez-Gaumé , G.Veneziano, N.Lambert and A.Sagnotti for several valuable discussions.

\section{$9 \quad$ Appendices}

\section{A Appendix A : Doubling trick, some useful correla- tion functions}

In order to deal with standard holomorphic conformal field theory propagators on the boundary of world sheet we might use the doubling trick. Having taken this trick, the world-sheet fields should be extended to the whole complex plane. Thus we must consider the following change of variables

$$
\tilde{X}^{\mu}(\bar{z}) \rightarrow D_{\nu}^{\mu} X^{\nu}(\bar{z}), \quad \tilde{\psi}^{\mu}(\bar{z}) \rightarrow D_{\nu}^{\mu} \psi^{\nu}(\bar{z}), \quad \tilde{\phi}(\bar{z}) \rightarrow \phi(\bar{z}), \quad \text { and } \quad \tilde{S}_{\alpha}(\bar{z}) \rightarrow M_{\alpha}{ }^{\beta} S_{\beta}(\bar{z})
$$

Notice that in the last relation left-moving spin operator gets replaced with the product of the constant $\mathrm{M}$ matrix and complex spin operator. The definitions of $D$ and $M$ matrix are :

$$
D=\left(\begin{array}{cc}
-1_{9-p} & 0 \\
0 & 1_{p+1}
\end{array}\right) \quad \text { and } \quad M_{p}=\left\{\begin{array}{cc}
\frac{ \pm i}{(p+1) !} \gamma^{a_{1}} \gamma^{a_{2}} \ldots \gamma^{a_{p+1}} \epsilon_{a_{1} \ldots a_{p+1}} & \text { for p even } \\
\frac{ \pm 1}{(p+1) !} \gamma^{a_{1}} \gamma^{a_{2}} \ldots \gamma^{a_{p+1}} \gamma_{11} \epsilon_{a_{1} \ldots a_{p+1}} & \text { for podd }
\end{array}\right.
$$

Now we are allowed to use just the holomorphic correlators for all fields $X^{\mu}, \psi^{\mu}, \phi$ as

$$
\begin{aligned}
\left\langle X^{\mu}(z) X^{\nu}(w)\right\rangle & =-\eta^{\mu \nu} \log (z-w) \\
\left\langle\psi^{\mu}(z) \psi^{\nu}(w)\right\rangle & =-\eta^{\mu \nu}(z-w)^{-1} \\
\langle\phi(z) \phi(w)\rangle & =-\log (z-w) .
\end{aligned}
$$


The Wick-like rule [21, 55] and [67] has been used to find out the correlation function between two spin operators and several fermion fields such that

$$
\begin{aligned}
\left\langle\psi^{\mu_{1}}\left(x_{1}\right) \ldots \psi^{\mu_{n}}\left(x_{n}\right) S_{\alpha}(z) S_{\beta}(\bar{z})\right\rangle= & \frac{1}{2^{n / 2}} \frac{(z-\bar{z})^{n / 2-5 / 4}}{\left|x_{1}-z\right| \ldots\left|x_{n}-z\right|}\left[\left(\Gamma^{\mu_{n} \ldots \mu_{1}} C^{-1}\right)_{\alpha \beta}\right. \\
& +\left\langle\left\langle\psi^{\mu_{1}}\left(x_{1}\right) \psi^{\mu_{2}}\left(x_{2}\right)\right\rangle\right\rangle\left(\Gamma^{\mu_{n} \ldots \mu_{3}} C^{-1}\right)_{\alpha \beta} \pm \text { perms } \\
& +\left\langle\left\langle\psi^{\mu_{1}}\left(x_{1}\right) \psi^{\mu_{2}}\left(x_{2}\right)\right\rangle\right\rangle\left\langle\left\langle\psi^{\mu_{3}}\left(x_{3}\right) \psi^{\mu_{4}}\left(x_{4}\right)\right\rangle\right\rangle\left(\Gamma^{\mu_{n} \ldots \mu_{5}} C^{-1}\right)_{\alpha \beta} \\
& \pm \text { perms }+\cdots]
\end{aligned}
$$

Note that the summation on all possible contractions must be assumed. $\Gamma^{\mu_{n} \ldots \mu_{1}}$ has to be a total antisymmetric matrix in terms of the gamma matrices. The Wick-like contraction is expressed as

$$
\begin{aligned}
\left\langle\left\langle\psi^{\mu}\left(x_{1}\right) \psi^{\nu}\left(x_{2}\right)\right\rangle\right\rangle & =\eta^{\mu \nu} \frac{\left(x_{1}-z\right)\left(x_{2}-\bar{z}\right)+\left(x_{2}-z\right)\left(x_{1}-\bar{z}\right)}{\left(x_{1}-x_{2}\right)(z-\bar{z})} \\
& =2 \eta^{\mu \nu} \frac{\operatorname{Re}\left[\left(x_{1}-z\right)\left(x_{2}-\bar{z}\right)\right]}{\left(x_{1}-x_{2}\right)(z-\bar{z})}
\end{aligned}
$$

Notice the fact that $x_{1}, x_{2}$ must be real. Applying (2) we can easily get the correlation function between two spin operators and one fermion field as

$$
I_{5}^{c}=<: S_{\alpha}\left(x_{4}\right): S_{\beta}\left(x_{5}\right): \psi^{c}\left(x_{3}\right):>=2^{-1 / 2} x_{45}^{-3 / 4}\left(x_{34} x_{35}\right)^{-1 / 2}\left(\gamma^{c} C^{-1}\right)_{\alpha \beta} .
$$

where $\Gamma^{\mu \nu}=\left(\gamma^{\mu} \gamma^{\nu}-\gamma^{\nu} \gamma^{\mu}\right) / 2$ and we have defined $x_{4} \equiv z=x+i y, x_{5} \equiv \bar{z}$ and $x_{i j}=x_{i}-x_{j}$.

Truly we were able to extend the Wick-like rule such that the correlation function of two spin operators and a number of mixed fermions and currents can be achieved [6, 23], provided the fact that one must remove Wick-like rule for two fermion fields $\psi \mathrm{s}$ in one current. Considering this point, we found consistent results in the absence and presence of current as follows

$$
\begin{aligned}
<: S_{\alpha}\left(x_{4}\right): S_{\beta}\left(x_{5}\right):> & =x_{45}^{-5 / 4} C_{\alpha \beta}^{-1}, \\
<: S_{\alpha}\left(x_{4}\right): S_{\beta}\left(x_{5}\right): \psi^{e} \psi^{f}\left(x_{1}\right):> & =-\frac{1}{2} x_{45}^{-1 / 4} x_{14}^{-1} x_{15}^{-1}\left(\Gamma^{e f} C^{-1}\right)_{\alpha \beta} .
\end{aligned}
$$

In the second formula of (5), we just removed Wick-like rule between two fermions in $x_{1}$ place and got consistent results . 


\section{B Appendix B: Some useful integrals for five-point functions}

To get some ideas let us mention the method that we have solved the integrals for five-point functions. We have gauge fixed by fixing the position of three open string operators and we are left with double integrals on $z, \bar{z}$. They are related to the $\mathrm{RR}$ closed string. Thus after gauge fixing we get the following double integrals:

$$
I=\int_{\mathcal{H}^{+}} d^{2} z|1-z|^{a}|z|^{b}(z-\bar{z})^{c}(z+\bar{z})^{d}
$$

where $d=0,1,2$ and $a, b, c$ should be computed in terms of the Mandelstam variables. Since we are talking about disk level amplitude the integrations must be done on upper half plane. The necessary conditions for these integrals must be taken into account as

$$
\begin{aligned}
& a+b+c \leq-2 \\
& a+b+d \leq-2
\end{aligned}
$$

To remove integrals on $x, y$ we may use the following definitions

$$
\begin{aligned}
|z|^{b} & =\frac{1}{\Gamma\left(-\frac{b}{2}\right)} \int_{0}^{\infty} d u u^{-\frac{b}{2}-1} e^{-u|z|^{2}}, \\
|1-z|^{a} & =\frac{1}{\Gamma\left(-\frac{a}{2}\right)} \int_{0}^{\infty} d s s^{-\frac{a}{2}-1} e^{-s|1-z|^{2}} .
\end{aligned}
$$

where $z=x+i y$

$$
\begin{gathered}
I_{y}=\int_{0}^{\infty} d y y^{c} e^{-(s+u) y^{2}}=\frac{\Gamma\left(\frac{1+c}{2}\right)}{2(s+u)^{\frac{1+c}{2}}} \\
F(\lambda)=\int_{-\infty}^{\infty} d x e^{-(s+u) x^{2}+2 \lambda x}=\sqrt{\frac{\pi}{s+u}} e^{\frac{\lambda^{2}}{s+u}} .
\end{gathered}
$$

Here we rewrite down the integration on $x$

$$
I_{x}=2^{d} \int_{-\infty}^{\infty} d x x^{d} e^{-s} e^{-(s+u) x^{2}+2 s x}=2^{d} e^{-s} \int_{-\infty}^{\infty} d x x^{d} e^{-(s+u)\left(x-\frac{s}{s+u}\right)^{2}+\frac{s^{2}}{s+u}} .
$$


so after all the integration on $x$ will be appeared as

$$
I_{x}=2^{d} e^{-\frac{u s}{s+u}} \int_{-\infty}^{\infty} d x x^{d} e^{-(s+u)\left(x-\frac{s}{s+u}\right)^{2}}=\left.e^{-s} \frac{d}{d^{d} \lambda} F(\lambda)\right|_{\lambda=s} .
$$

$d=n, \quad n \in Z$,

$$
I_{x}=2^{d} e^{-\frac{u s}{s+u}} \frac{\sqrt{\pi}}{(s+u)^{\frac{1}{2}}}\left\{\begin{array}{cl}
1 & , d=0 \\
\frac{s}{(s+u)} & , d=1
\end{array}\right.
$$

For simplicity we just do the integration for $d=0$ and finally we show our results for $d=1,2$. So for $d=0$ after replacing those steps mentioned above and doing the integrals over $x, y$, collecting them and replacing in the general integration on $I$, we will have

$$
I=\frac{\sqrt{\pi}(2 i)^{c+1} 2^{0} \Gamma\left(\frac{1+c}{2}\right)}{2 \Gamma\left(\frac{-a}{2}\right) \Gamma\left(\frac{-b}{2}\right)} \int_{0}^{\infty} \int_{0}^{\infty} \frac{d s d u}{(s+u)^{1+c / 2}} u^{-b / 2-1} s^{-a / 2-1} e^{-\frac{u s}{s+u}}
$$

We might use the following change of variables

$$
s=\frac{x}{t}, \quad u=\frac{x}{1-t}, \quad d s d u=J d x d t=\frac{x d x d t}{(t(1-t))^{2}}
$$

Replacing the change of variables in the Jacobian, we find

$$
\begin{gathered}
I=\frac{\pi^{1 / 2}(2 i)^{c+1} \Gamma\left(\frac{1+c}{2}\right)}{2 \Gamma\left(\frac{-a}{2}\right) \Gamma\left(\frac{-b}{2}\right)} \int_{0}^{\infty} d x e^{-x} x^{\frac{-4-(a+b+c)}{2}} \int_{0}^{1} d t t^{(c+a) / 2}(1-t)^{(c+b) / 2}, \\
I=(2 \imath)^{c} \pi \frac{\Gamma\left(1+\frac{b+c}{2}\right) \Gamma\left(1+\frac{a+c}{2}\right) \Gamma\left(-1-\frac{a+b+c}{2}\right) \Gamma\left(\frac{1+c}{2}\right)}{\Gamma\left(-\frac{a}{2}\right) \Gamma\left(-\frac{b}{2}\right) \Gamma\left(2+c+\frac{a+b}{2}\right)} .
\end{gathered}
$$

The following relations have been used

$$
\int_{0}^{1} d x x^{\beta-1}(1-x)^{\alpha-1}=\frac{\Gamma(\alpha) \Gamma(\beta)}{\Gamma(\alpha+\beta)}, \quad \Gamma(z)=(z-1) !
$$

Eventually we obtain the result for $d=1$ as

$$
I=(2 \imath)^{c} 2 \pi \frac{\Gamma\left(2+\frac{b+c}{2}\right) \Gamma\left(1+\frac{a+c}{2}\right) \Gamma\left(-1-\frac{a+b+c}{2}\right) \Gamma\left(\frac{1+c}{2}\right)}{\Gamma\left(-\frac{a}{2}\right) \Gamma\left(-\frac{b}{2}\right) \Gamma\left(3+c+\frac{a+b}{2}\right)},
$$

Therefore one can write them down in a closed form as

$$
\int d^{2} z|1-z|^{a}|z|^{b}(z-\bar{z})^{c}(z+\bar{z})^{d}=(2 i)^{c} 2^{d} \pi \frac{\Gamma\left(1+d+\frac{b+c}{2}\right) \Gamma\left(1+\frac{a+c}{2}\right) \Gamma\left(-1-\frac{a+b+c}{2}\right) \Gamma\left(\frac{1+c}{2}\right)}{\Gamma\left(-\frac{a}{2}\right) \Gamma\left(-\frac{b}{2}\right) \Gamma\left(2+c+d+\frac{a+b}{2}\right)} .
$$


The above result is valid For $d=0,1$ 68]. Applying the same method for $d=2$, one sets 23 .

$$
\int d^{2} z|1-z|^{a}|z|^{b}(z-\bar{z})^{c}(z+\bar{z})^{d}=(2 i)^{c} 2^{d} \pi \frac{J_{1}+J_{2}}{\Gamma\left(-\frac{a}{2}\right) \Gamma\left(-\frac{b}{2}\right) \Gamma\left(d+2+c+\frac{a+b}{2}\right)} .
$$

where

$$
\begin{aligned}
& J_{1}=\frac{1}{2} \Gamma\left(d+\frac{b+c}{2}\right) \Gamma\left(d+\frac{a+c}{2}\right) \Gamma\left(-d-\frac{a+b+c}{2}\right) \Gamma\left(\frac{1+c}{2}\right) \\
& J_{2}=\Gamma\left(d+1+\frac{b+c}{2}\right) \Gamma\left(1+\frac{a+c}{2}\right) \Gamma\left(-1-\frac{a+b+c}{2}\right) \Gamma\left(\frac{1+c}{2}\right) .
\end{aligned}
$$

\section{References}

[1] J. Polchinski, "Dirichlet-Branes and Ramond-Ramond Charges," Phys. Rev. Lett. 75, 4724 (1995) arXiv:hep-th/9510017.

[2] E. Witten, "Bound states of strings and p-branes," Nucl. Phys. B 460,335 (1996) arXiv:hep-th/9510135.

[3] J. Polchinski, "Lectures on D-branes," arXiv:hep-th/9611050] ; C. P. Bachas, "Lectures on D-branes," arXiv:hep-th/9806199.

[4] R. C. Myers, "Dielectric-branes," JHEP 9912, 022 (1999) arXiv:hep-th/9910053.

[5] W. Taylor and M. Van Raamsdonk, "Multiple Dp-branes in weak background fields," Nucl. Phys. B 573,703 (2000) arXiv:hep-th/9910052.

[6] E. Hatefi, "On effective actions of BPS branes and their higher derivative corrections," JHEP 1005, 080 (2010) arXiv:1003.0314 [hep-th]].

[7] E. Hatefi and I. Y. Park, "More on closed string induced higher derivative interactions on D-branes," Phys. Rev. D 85, 125039 (2012) arXiv:1203.5553 [hep-th]].

[8] E. Hatefi and I. Y. Park, "Universality in all-order $\alpha^{\prime}$ corrections to BPS/non-BPS brane world volume theories," Nucl. Phys. B 864, 640 (2012) arXiv:1205.5079 [hepth]].

[9] E. Hatefi, "Three Point Tree Level Amplitude in Superstring Theory," Nucl. Phys. Proc. Suppl. 216, 234 (2011) arXiv:1102.5042 [hep-th]]; M. R. Garousi and 
R. C. Myers, "World volume potentials on D-branes," JHEP 0011, 032 (2000) hep-th/0010122.

[10] M. Li, "Boundary states of D-branes and dy-strings," Nucl. Phys. B460, 351 (1996) arXiv:hep-th/9510161 ; M. R. Douglas, "Branes within branes," arXiv:hep-th/9512077] ; M. B. Green, J. A. Harvey and G. W. Moore, "I-brane inflow and anomalous couplings on D-branes," Class. Quant. Grav. 14, 47 (1997) arXiv:hep-th/9605033.

[11] A. Sen, "NonBPS states and Branes in string theory," hep-th/9904207.

[12] P. Horava, "Type IIA D-branes, K theory, and matrix theory," Adv. Theor. Math. Phys. 2, 1373 (1999) hep-th/9812135].

[13] O. Bergman and M. R. Gaberdiel, "Stable nonBPS D particles," Phys. Lett. B 441, 133 (1998) hep-th/9806155; A. Sen, "SO(32) spinors of type I and other solitons on brane - anti-brane pair," JHEP 9809, 023 (1998) hep-th/9808141]; A. Sen, "BPS D-branes on nonsupersymmetric cycles," JHEP 9812, 021 (1998) hep-th/9812031; M. Frau, L. Gallot, A. Lerda and P. Strigazzi, "Stable nonBPS D-branes in type I string theory," Nucl. Phys. B 564, 60 (2000) [hep-th/9903123].

[14] E. Witten, "D-branes and K theory," JHEP 9812, 019 (1998) hep-th/9810188; O. Bergman, E. G. Gimon and P. Horava, "Brane transfer operations and T duality of nonBPS states," JHEP 9904, 010 (1999) hep-th/9902160.

[15] A. Sen, "Stable nonBPS states in string theory," JHEP 9806, 007 (1998) hep-th/9803194.

[16] A. Sen, "Tachyon dynamics in open string theory," Int. J. Mod. Phys. A 20, 5513 (2005) hep-th/0410103.

[17] P. Kraus and F. Larsen, "Boundary string field theory of the D anti-D system," Phys. Rev. D 63, 106004 (2001) [hep-th/0012198].

[18] T. Takayanagi, S. Terashima and T. Uesugi, "Brane - anti-brane action from boundary string field theory," JHEP 0103, 019 (2001) hep-th/0012210].

[19] C. Kennedy and A. Wilkins, "Ramond-Ramond couplings on Brane - anti-Brane systems," Phys. Lett. B 464, 206 (1999) [hep-th/9905195]. 
[20] G. Roepstorff, "Superconnections and the Higgs field," J. Math. Phys. 40, 2698 (1999) hep-th/9801040; D.Quillen, Topology 24(1995) 8995;N.Berline,E.Getzler,M.Vergne, Heat Kernels and Dirac Operators,Springer,1991

[21] M. R. Garousi and E. Hatefi, "On Wess-Zumino terms of Brane-Antibrane systems," Nucl. Phys. B 800, 502 (2008) arXiv:0710.5875 [hep-th]].

[22] M. R. Garousi, "On Wess-Zumino terms of non-BPS D-branes and their higher derivative corrections," Nucl. Phys. B 809, 525 (2009) [arXiv:0802.2784 [hep-th]].

[23] M. R. Garousi and E. Hatefi, "More on WZ action of non-BPS branes," JHEP 0903, 008 (2009) [arXiv:0812.4216 [hep-th]].

[24] M. R. Garousi, "Tachyon couplings on nonBPS D-branes and Dirac-Born-Infeld action," Nucl. Phys. B 584, 284 (2000) hep-th/0003122.

[25] M. R. Garousi, "On modified tachyon DBI action," Nucl. Phys. B 817, 252 (2009) arXiv:0811.4334 [hep-th]].

[26] P. -J. De Smet and J. Raeymaekers, "The Tachyon potential in Witten's superstring field theory," JHEP 0008, 020 (2000) hep-th/0004112.

[27] E. Hatefi, " in preparation,"

[28] J. Polchinski, "Combinatorics of boundaries in string theory," Phys. Rev. D50,6041 (1994) arXiv:hep-th/9407031; J. Polchinski, S. Chaudhuri and C. V. Johnson, "Notes on D-Branes," arXiv:hep-th/9602052.

[29] I.Y.Park, "Scattering on D3-branes," Phys. Lett. B660, 583 (2008) arXiv:0708.3452[hep-th]] I. Y. Park, "Open string engineering of D-brane geometry," JHEP 0808, 026 (2008) [arXiv:0806.3330[hep-th]].

[30] A. Hashimoto and I. R. Klebanov, "Scattering of strings from D-branes," Nucl. Phys. Proc. Suppl. 55B, 118 (1997) arXiv:hep-th/9611214] ; A. Hashimoto and I. R. Klebanov , "Decay of excited D-branes," Phys. Lett. B381, 437 (1996) arXiv:hep-th/9604065 ; I. R. Klebanov and L. Thorlacius, "The Size of p-branes," Phys. Lett. B371, 51 (1996) arXiv:hep-th/9510200 ; S.S. Gubser, A. Hashimoto, I.R. Klebanov, and J.M. Maldacena, "Gravitational lensing by p-branes," Nucl. Phys. B472, 231 (1996) arXiv:hep-th/9601057 ; C. Bachas, "D-Brane Dynamics," Phys. 
Lett. B374, 37 (1996) arXiv:hep-th/9511043 ; J. Polchinski, "String duality: A colloquium," Rev. Mod. Phys. 68, 1245 (1996) arXiv:hep-th/9607050]; W. Taylor, "Lectures on D-branes, gauge theory and M(atrices)," arXiv:hep-th/9801182; C. Vafa, "Lectures on strings and dualities," arXiv:hep-th/9702201] ; M. Billo, M. Frau, F. Lonegro and A. Lerda, "N =1/2 quiver gauge theories from open strings with R-R fluxes," JHEP 0505,047 (2005) arXiv:hep-th/0502084] ; M. Billo, P. Di Vecchia, M. Frau, A. Lerda, I. Pesando, R. Russo and S. Sciuto, "Microscopic string analysis of the D0-D8 brane system and dual R-R states," Nucl. Phys. B 526,199 (1998) arXiv:hep-th/9802088; R. H. Boels, D. Marmiroli and N. A. Obers, "On-shell Recursion in String Theory," JHEP 1010, 034 (2010) [arXiv:1002.5029 [hep-th]]; A. Sagnotti and M. Taronna, "String Lessons for Higher-Spin Interactions," Nucl. Phys. B 842, 299 (2011) arXiv:1006.5242 [hep-th]]; E. Hatefi, A. J. Nurmagambetov and I. Y. Park, "Near-Extremal Black-Branes with n*3 Entropy Growth," Int. J. Mod. Phys. A 27, 1250182 (2012) [arXiv:1204.6303 [hep-th]]; E. Hatefi, A. J. Nurmagambetov and I. Y. Park, " $N^{3}$ entropy of $M 5$ branes from dielectric effect," Nucl. Phys. B 866, 58 (2013) [arXiv:1204.2711 [hep-th]]; E. Hatefi, A. J. Nurmagambetov and I. Y. Park, "ADM reduction of IIB on $H^{p, q}$ to dS braneworld," arXiv:1210.3825 [hep-th] to appear in JHEP; E. Hatefi, "Shedding light on new Wess-Zumino couplings with their corrections to all orders in alpha-prime," JHEP 1304, 070 (2013) [arXiv:1211.2413 [hep-th]]; E. Hatefi, "On D-brane anti D-brane effective actions and their corrections to all orders in alpha-prime," arXiv:1211.5538 [hep-th]; E. Hatefi, "Closed string Ramond-Ramond proposed higher derivative interactions on fermionic amplitudes in IIB," arXiv:1302.5024 [hep-th]; E. Hatefi, "All order $\alpha^{\prime}$ higher derivative corrections to non-BPS branes of type IIB Super string theory," arXiv:1304.3711 [hep-th]; E. Dudas, J. Mourad and A. Sagnotti, "Charged and uncharged D-branes in various string theories," Nucl. Phys. B 620, 109 (2002) hep-th/0107081.

[31] R. Britto, F. Cachazo and B. Feng, "New recursion relations for tree amplitudes of gluons," Nucl. Phys. B 715, 499 (2005) hep-th/0412308].

[32] N. Arkani-Hamed and J. Kaplan, "On Tree Amplitudes in Gauge Theory and Gravity," JHEP 0804, 076 (2008) [arXiv:0801.2385 [hep-th]]. 
[33] C. Cheung, "On-Shell Recursion Relations for Generic Theories," JHEP 1003, 098 (2010) arXiv:0808.0504 [hep-th]].

[34] C. Cheung, D. O'Connell and B. Wecht, "BCFW Recursion Relations and String Theory," JHEP 1009, 052 (2010) [arXiv:1002.4674 [hep-th]].

[35] A. Fotopoulos and N. Prezas, "Pomerons and BCFW recursion relations for strings on D-branes," Nucl. Phys. B 845, 340 (2011) [arXiv:1009.3903 [hep-th]].

[36] M. Gutperle and A. Strominger, "Space - like branes," JHEP 0204, 018 (2002) hep-th/0202210.

[37] A. Sen, "Rolling tachyon," JHEP 0204, 048 (2002) [hep-th/0203211]. F. Larsen, A. Naqvi and S. Terashima, "Rolling tachyons and decaying branes," JHEP 0302, 039 (2003) hep-th/0212248]. S. -J. Rey and S. Sugimoto, "Rolling of modulated tachyon with gauge flux and emergent fundamental string," Phys. Rev. D 68, 026003 (2003) hep-th/0303133]; N. D. Lambert, H. Liu and J. M. Maldacena, "Closed strings from decaying D-branes," JHEP 0703, 014 (2007) hep-th/0303139.

[38] A. Sen, "Supersymmetric world volume action for nonBPS D-branes," JHEP 9910, 008 (1999) hep-th/9909062];

[39] E. A. Bergshoeff, M. de Roo, T. C. de Wit, E. Eyras and S. Panda, "T duality and actions for nonBPS D-branes," JHEP 0005, 009 (2000) hep-th/0003221; J. Kluson, "Proposal for nonBPS D-brane action," Phys. Rev. D 62, 126003 (2000) hep-th/0004106.

[40] A. Sen, "Field theory of tachyon matter," Mod. Phys. Lett. A 17, 1797 (2002) hep-th/0204143.

[41] R. Casero, E. Kiritsis and A. Paredes, "Chiral symmetry breaking as open string tachyon condensation," Nucl. Phys. B 787, 98 (2007) hep-th/0702155 [HEP-TH]]; O. Bergman, S. Seki and J. Sonnenschein, "Quark mass and condensate in HQCD," JHEP 0712, 037 (2007) arXiv:0708.2839 [hep-th]]. A. Dhar and P. Nag, "SakaiSugimoto model, Tachyon Condensation and Chiral symmetry Breaking," JHEP 0801, 055 (2008) [arXiv:0708.3233 [hep-th]]. 
[42] G. R. Dvali and S. H. H. Tye, "Brane inflation," Phys. Lett. B 450 (1999) 72 hep-ph/9812483]; G. R. Dvali, Q. Shafi and S. Solganik, "D-brane inflation," hep-th/0105203; C. P. Burgess, M. Majumdar, D. Nolte, F. Quevedo, G. Rajesh and R. -J. Zhang, "The Inflationary brane anti-brane universe," JHEP 0107, 047 (2001) hep-th/0105204]; S. Kachru, R. Kallosh, A. D. Linde, J. M. Maldacena, L. P. McAllister and S. P. Trivedi, "Towards inflation in string theory," JCAP 0310, 013 (2003) hep-th/0308055.

[43] T. Banks and L. Susskind, "Brane - anti-brane forces," hep-th/9511194.

[44] A. Sen, "Stable nonBPS bound states of BPS D-branes," JHEP 9808, 010 (1998) hep-th/9805019.

[45] S. P. de Alwis, "Tachyon condensation in rotated brane configurations," Phys. Lett. B 461, 329 (1999) hep-th/9905080.

[46] A. Sen, "Tachyon condensation on the brane anti-brane system," JHEP 9808, 012 (1998) hep-th/9805170].

[47] L. Kofman, A. D. Linde and A. A. Starobinsky, "Towards the theory of reheating after inflation," Phys. Rev. D 56, 3258 (1997) hep-ph/9704452; R. H. Brandenberger, A. R. Frey and L. C. Lorenz, "Entropy fluctuations in brane inflation models," Int. J. Mod. Phys. A 24, 4327 (2009) [arXiv:0712.2178 [hep-th]].

[48] S. H. S. Alexander, "Inflation from D - anti-D-brane annihilation," Phys. Rev. D 65, 023507 (2002) hep-th/0105032; T. Padmanabhan, Phys. Rev. D 66, 021301 (2002) hep-th/0204150; A. V. Frolov, L. Kofman and A. A. Starobinsky, Phys. Lett. B 545, 8 (2002) hep-th/0204187]; L. Kofman and A. D. Linde, JHEP 0207, 004 (2002) hep-th/0205121]; G. Shiu, S. H. H. Tye and I. Wasserman, Phys. Rev. D 67, 083517 (2003) hep-th/0207119] F. Quevedo, Class. Quant. Grav. 19, 5721 (2002) hep-th/0210292; J. M. Cline, H. Firouzjahi and P. Martineau, "Reheating from tachyon condensation," JHEP 0211, 041 (2002) hep-th/0207156.

[49] A. Sen, "Tachyon matter," JHEP 0207, 065 (2002) hep-th/0203265.

[50] E. Hatefi, To appear

[51] D. Kutasov, M. Marino and G. W. Moore, "Remarks on tachyon condensation in superstring field theory," hep-th/0010108. 
[52] A. A. Tseytlin, "Sigma model approach to string theory effective actions with tachyons," J. Math. Phys. 42, 2854 (2001) hep-th/0011033.

[53] J. A. Minahan and B. Zwiebach, "Effective tachyon dynamics in superstring theory," JHEP 0103, 038 (2001) hep-th/0009246.

[54] S. Stieberger, "Open and Closed vs. Pure Open String Disk Amplitudes," arXiv:0907.2211 [hep-th]; R. Medina, F. T. Brandt and F. R. Machado, "The Open superstring five point amplitude revisited," JHEP 0207, 071 (2002) hep-th/0208121.

[55] H. Liu and J. Michelson, "*-trek 3: The Search for Ramond-Ramond couplings," Nucl. Phys. B 614, 330 (2001) hep-th/0107172].

[56] M. Billo, B. Craps and F. Roose, "Ramond-Ramond couplings of nonBPS D-branes," JHEP 9906, 033 (1999) hep-th/9905157.

[57] C. M. Hull, "Matrix theory, U duality and toroidal compactifications of M theory," JHEP 9810, 011 (1998) [hep-th/9711179]; H. Dorn, "NonAbelian gauge field dynamics on matrix D-branes," Nucl. Phys. B 494, 105 (1997) hep-th/9612120.

[58] M. R. Douglas, "D-branes in curved space," Adv. Theor. Math. Phys. 1, 198 (1998) hep-th/9703056]; M. R. Douglas, "D-branes and matrix theory in curved space," Nucl. Phys. Proc. Suppl. 68, 381 (1998) hep-th/9707228]; M. R. Douglas, A. Kato and H. Ooguri, "D-brane actions on Kahler manifolds," Adv. Theor. Math. Phys. 1, 237 (1998) |hep-th/9708012.

[59] A. Sen, "Descent relations among bosonic D-branes," Int. J. Mod. Phys. A 14, 4061 (1999) [hep-th/9902105]; A. Recknagel and V. Schomerus, "Boundary deformation theory and moduli spaces of D-branes," Nucl. Phys. B 545, 233 (1999) hep-th/9811237]; C. G. Callan, I. R. Klebanov, A. W. W. Ludwig and J. M. Maldacena, "Exact solution of a boundary conformal field theory," Nucl. Phys. B 422, 417 (1994) |hep-th/9402113.

[60] M. R. Garousi and H. Golchin, "On higher derivative corrections of the tachyon action," Nucl. Phys. B 800, 547 (2008) [arXiv:0801.3358 [hep-th]].

[61] E. Hatefi, " in preparation," 
[62] E. Hatefi "On six point functions and fundamental problems"

[63] A. A. Tseytlin, "On nonAbelian generalization of Born-Infeld action in string theory," Nucl. Phys. B 501, 41 (1997) hep-th/9701125].

[64] A. Hashimoto and W. Taylor, "Fluctuation spectra of tilted and intersecting D-branes from the Born-Infeld action," Nucl. Phys. B 503, 193 (1997) hep-th/9703217; P. Bain, "On the nonAbelian Born-Infeld action," hep-th/9909154.

[65] L. Cornalba, "Corrections to the Abelian Born-Infeld action arising from noncommutative geometry," JHEP 0009, 017 (2000) hep-th/9912293]; L. Cornalba, "On the general structure of the nonAbelian Born-Infeld action," Adv. Theor. Math. Phys. 4, 1259 (2002) [hep-th/0006018]; Y. Okawa, "Derivative corrections to DiracBorn-Infeld Lagrangian and noncommutative gauge theory," Nucl. Phys. B 566, 348 (2000) [hep-th/9909132].

[66] M. R. Douglas and C. M. Hull, "D-branes and the noncommutative torus," JHEP 9802, 008 (1998) hep-th/9711165]; A. Connes, M. R. Douglas and A. S. Schwarz, "Noncommutative geometry and matrix theory: Compactification on tori," JHEP 9802, 003 (1998) hep-th/9711162]; N. Seiberg and E. Witten, "String theory and noncommutative geometry," JHEP 9909, 032 (1999) [hep-th/9908142].

[67] V. A. Kostelecky, O. Lechtenfeld, W. Lerche, S. Samuel and S. Watamura, "Conformal Techniques, Bosonization and Tree Level String Amplitudes," Nucl. Phys. B 288, 173 (1987).

[68] A. Fotopoulos, "On (alpha-prime)**2 corrections to the D-brane action for nongeodesic world volume embeddings," JHEP 0109, 005 (2001) [hep-th/0104146]. 